\title{
Rassegna bibliografica
}

\section{Educare gli italiani}

Giuseppe Ricuperati, Storia della Scuola in Italia, Brescia, La Scuola, 2015, pp. 410 , euro 23,50 .

Come ci ha insegnato Benedetto Croce, ogni storia è sempre storia contemporanea e questo è ancor più vero per la storia della scuola, ma a maggior ragione per quella dell'università, quando è praticata dagli storici come "secondo mestiere". In tale chiave possiamo leggere questo denso volume di Giuseppe Ricuperati che riprende e rifonde vari suoi scritti che l'opportunità editoriale presenta come se si trattasse di una sintesi manualistica. Lo stesso Ricuperati si era a più riprese cimentato sia in grandi opere come la Letteratura italiana Einaudi e soprattutto la Storia d'Italia e nella Storia dell'Italia repubblicana sempre di Einaudi e anche in forma di antologia di docenti (C. Canestri, G. Ricuperati, La scuola in Italia dalla legge Casati ad oggi, Torino, Loescher, 1976). Un simile impegno era parallelo a quello maggiore di simpatetico studioso dei riformatori illuministi e portava inevitabilmente a partire dai progetti e dai momenti di riforma piuttosto che dai tempi lunghi dell'apparente immobilismo della realtà quotidiana dell'amministrazione.

Si tratta di una articolata riflessione storiografica in cui i vari momenti sono letti come riforme mancate o al più come riforme inadeguate. La vicenda postunitaria è quindi soprattutto quella della impossibile revisione della Casati malgrado gli sforzi di un ministro del calibro di Francesco De Sanctis. La forte simpateticità di Ricuperati per la cultura settecentesca lo porta quasi inconsapevolmente a mettere quasi del tutto fra parentesi la cultura pedagogica dell'età del Risorgimento che, nelle sue diverse componenti e anche nei suoi protagonisti, è animata da esperienze e scritti in materia educativa e dunque trova nella Casati la dimensione scolastica della soluzione unitaria e monarchicoliberale.

La riforma Gentile viene esaminata a partire da un intervento di autodifesa di Gentile; si tratta certamente di una lettura che sottolinea la continuità con la politica del fascismo, a differenza di quanto ha spesso fatto la storiografia pedagogica di matrice codignoliana (da Borghi a Tomasi e a Santoni Rugiu e i loro allievi). Rimane abbastanza in ombra il riformismo "minore" dell'età giolittiana, le leggi Orlando e Daneo-Credaro e soprattutto l'impegno per la scuola elementare di cui emblematica rimane l'inchiesta coordinata da Camillo Corradini, funzionario particolarmente vicino a Giolitti.

Le vicende della scuola e dell'università in periodo fascista sono ricostruite in un'ottica di storia politica non distante da 
quella praticata negli anni Settanta e Ottanta dai suoi colleghi torinesi come Nicola Tranfaglia. Affermare che la scuola italiana rimanga ancor oggi quella di Gentile significa implicitamente non solo relativizzare radicalmente le leggi degli ultimi vent'anni ma soprattutto lasciare del tutto sullo sfondo la dimensione quantitativa che è il nocciolo di quello definito da Marcello Dei come cambiamento senza riforma (La scuola secondaria superiore negli ultimi trent'anni, in S. Soldani, G. Turi, Fare gli Italiani. Scuola e cultura nell'Italia contemporanea, Bologna, Il Mulino, 1993, vol. II, pp. 87-127) in termini di massificazione della secondaria superiore.

Il secondo dopoguerra viene letto come "ricostruzione difficile" mettendo in penombra l'impegno per la ricostruzione materiale delle scuole e la portata innovativa del dettato costituzionale che anche in ambito scolastico è una rivoluzione promessa, per usare la classica espressione di Piero Calamandrei. Lo storico torinese è forse ingeneroso verso la media unica mentre nella narrazione ulteriore il testo si intreccia con la sua impegnata biografia che assolutizza una esperienza nel complesso relativamente privilegiata come quella della scuola e della università piemontesi che videro per esempio negli Irssae un reale luogo di contatto tra mondo della scuola e cultura accademica. Interessanti le pagine in cui ripercorre il suo impegno per la didattica della storia, certamente una delle pagine più feconde del lungo sessantotto subalpino (G. Ricuperati, Clio e il centauro Chirone. Interventi sull'insegnamento della storia, Bruno Mondadori, 1989 e Id., Apologia di un mestiere difficile. Problemi, insegnamenti e responsabilità della storia, Roma-Bari, Laterza, 2005) ed evoca il suo impegno come autore di fortunati manuali scolastici e universitari. La partecipe cronaca si estende fino ai nostri giorni con pagine di perplessa attesa rispetto ai provvedimenti nel frattempo appena entrati in vigore.

Lo storico della scuola in senso accademico deve rilevare come la storiogra- fia specifica sia stata talvolta non del tutto valorizzata, basti pensare alla monografia di Daria Gabusi, La svolta democratica nell'istruzione italiana. Luigi Gui e la politica scolastica del centro-sinistra (1962-1968), Brescia, La Scuola, 2010 e ai repertori diretti da Chiosso sull'editoria Teseo (Tipografi editori scolastici educativi dell'Ottocento), a cura di G. Chiosso, Milano, Bibliografica, 2003 e Teseo '900 Editori scolastico-educativi del primo Novecento, a cura di G. Chiosso, Milano, Bibliografica, 2008 e sui periodici scolastici La stampa pedagogica e scolastica in Italia 1820-1943, a cura di G. Chiosso, Brescia, La Scuola, 1997.

L'appassionata conclusione esplicita come lo storico torinese ritenga che "scuola, università e ricerca sono anche l'anima di una appartenenza non solo mia, ma anche di quanti ho formato, soprattutto, ho visto ogni giorno salvare la scuola, come atto costituzionale colto e competente, fieri di farlo senza essere del tutto riconosciuti" (p. 396). La collocazione editoriale è essa stessa un fatto rilevante per la classificazione pedagogica del volume ma anche per l'ispirazione dell'editore circostanze che testimoniano come ormai "storia della scuola" e "storia" siano una cosa sola e come Chiesa e mondo siano una storia sola.

Angelo Gaudio

Alberto Alberti, La scuola della Repubblica. Un ideale non realizzato, Roma, Anicia, 2016, pp. 366, euro 28.

Stereotipi e luoghi comuni hanno accompagnato da sempre il dibattito pubblico sulla scuola. Per superare le genericità, individuare i problemi strutturali e i punti di forza del nostro sistema di istruzione, c'è bisogno della prospettiva storica. Il volume di Alberti si distingue nella ormai vasta bibliografia sulla storia della scuola italiana per la capacità dell'autore di contestualizzare il discorso, "con riguardo non solo alle idee più meditate, più apprezzabili o più diffuse, ma an- 
che alle esigenze economiche e sociali e ai problemi quotidiani” (p. 102). La scuola della Repubblica è un libro "militante", animato da una passione civile maturata nell'impegno dell'autore come maestro, pedagogista, scrittore, dirigente scolastico. Questa esperienza gli consente di cogliere e descrivere il divario che spesso si apre fra la norma e la pratica scolastica, in base alla convinzione che "ogni documento programmatico va giudicato per il suo impatto sulla situazione reale" (p. 123). La narrazione storica è preceduta da quadri che intrecciano le vicende della scuola all'evoluzione sociale e politica italiana ed europea. Il volume presenta inoltre una serie di box, che evidenziano la dimensione diacronica di temi quali la dispersione scolastica, la formazione dei docenti, il ruolo delle autonomie locali, la valutazione, insieme con le impietose statistiche dell'Istat sulla percentuale di diplomati e laureati, che conosce incrementi lentissimi. Emergono dalla lettura alcuni mali storici del nostro sistema: la preminenza della cultura umanistica rispetto a quella scientifica, tecnica, professionale; la mancata riforma complessiva della scuola secondaria; le innovazioni scolastiche introdotte per via amministrativa anziché parlamentare e legislativa. Infine il volume presenta un'appendice sulla legislazione scolastica, dal regio decreto Casati del 1859 fino al 2011 e la "Raccomandazione" europea del 2006 sulle competenze chiave per l'apprendimento.

Nel ripercorrere le vicende della scuola italiana, Alberti applica un criterio interpretativo esplicitato dal sottotitolo del volume: Un ideale non realizzato. $\mathrm{Ci}$ sono, infatti, due tendenze, più che realtà compiute: una è quella della "scuola della Repubblica", la scuola del "non uno di meno", volta ad eliminare le disuguaglianze di origine. L'altra è la "scuola monarchica", un meccanismo di riproduzione delle divisioni sociali, compresi privilegi ed esclusioni. Il dissidio e l'intreccio fra i due modelli caratterizzano la storia repubblicana.
Il sistema scolastico italiano è nato e si è consolidato, fra lo Stato liberale e quello fascista, secondo principi monarchici: il centralismo, l'imposizione dell'autorità, l'impostazione elitaria (lo slogan "poche scuole ma buone"). Alla base è presente l'idea della separazione dei corsi di studi secondo il ceto di provenienza: la scuola superiore per i privilegiati, la scuola dell'obbligo per il popolo. Dopo la Seconda guerra mondiale, il modello monarchico entra in crisi. Per Alberti, il periodo che va dalla caduta del fascismo alla Liberazione rappresenta un "laboratorio": mentre nell'Italia settentrionale le Repubbliche partigiane mettono al primo posto dei loro programmi la scuola, nell'Italia meridionale il governo militare alleato diffonde il credo pedagogico democratico di Dewey (incontrando immediatamente l'ostilità dei settori più conservatori della società). Nella Costituzione la scuola, "aperta a tutti" (art. 34), neppure nominata nello Statuto Albertino, entra fra $i$ tratti fondanti della Repubblica. Negli anni del centrismo, le vie indicate dalla Costituzione sono messe in secondo piano; ci si limita a interventi di manutenzione, mentre le scuole private continuano a svolgere una funzione pubblica in sostituzione dello Stato.

Fra gli anni Sessanta e Settanta, come avviene in altri campi, si sommano molti elementi di discontinuità nel mondo laico come in quello cattolico: si diffondono scuole "altre" rispetto a quelle ufficiali, prima fra tutte la scuola di Barbiana di don Milani; pedagogisti e intellettuali discutono sulle riviste, genitori e insegnanti conoscono un nuovo protagonismo. Frutti duraturi di questa stagione sono la scuola media unica (1962), la scuola materna statale (1968), il tempo pieno alle elementari (1971), l'integrazione dei “diversi" (legge 517 del 1977), che insieme contribuiscono a tracciare il volto di una "scuola della Repubblica". Fra le riflessioni dell'autore, interessante è quella sul "tempo pieno" alle elementari: non una giornata di scuola più lunga, ma un modello pedagogico e educativo, secondo il quale l'apprendimen- 
to avviene non solo attraverso la comunicazione linguistica formalizzata, ma per molteplici canali (ricerca, attività, lavori di gruppo) che necessitano di un tempo ampio e disteso.

Nei decenni successivi, la spinta innovativa subisce un netto ridimensionamento. L'avvicendarsi di governi e ministri ognuno con la propria riforma, annunciata e mai conclusa, trasforma la politica scolastica della "seconda Repubblica" in una "tela di Penelope", la cui trama nasconde un netto taglio di risorse pubbliche. Permane l'autonomia scolastica (legge del 2001), chiave di volta di una "scuola della Repubblica", aperta al territorio, condivisa con gli studenti e i genitori. Eppure oggi, osserva Alberti, l'autonomia si presenta alle famiglie sotto forma di richieste di contributi, la scuola appare come un "progettificio", il ministero diffonde minuziose disposizioni didattiche mentre controlla in modo centralistico le risorse destinate alle scuole. Ciò che manca alla scuola italiana, in conclusione, è una "cultura dell'autonomia", nella quale tutti si sentano parte di un progetto condiviso, "come un canto popolare che si può cantare con efficacia e soddisfazione, anche quando non si sanno tutte le parole" (p. 275). Alle prese con la cosiddetta "Buona scuola", è difficile immaginare quale sarà il canto da intonare tutti insieme, dirigenti, insegnanti, studenti.

Irma Staderini

\section{Quando l'Italia è mafiosa}

Manoela Patti, La mafia alla sbarra. I processi fascisti a Palermo, Prefazione di Salvatore Lupo, Istituto Poligrafico Europeo, Palermo, 2014, pp. XII-255, euro 15.

Per rivelarsi agli occhi dello storico, la mafia necessita di un'azione antimafia che ne indaghi le trame e ne restituisca, nei limiti del possibile, un ritratto dei protagonisti e delle dinamiche di sviluppo. Compito dello storico è quello di mettersi sulle trac- ce delle fonti messe a disposizione da tale lavoro di contrasto e giungere a una sintesi ricostruttiva che si fondi su un'attenta interpretazione di un materiale che offre, naturalmente, ampi margini di incertezza. Le larghe operazioni antimafia avviate dalla seconda metà degli anni venti dal regime fascista, poi riprese sotto forme differenti negli anni Trenta, con il loro lascito documentario offrono, in tal senso, strumenti conoscitivi essenziali per guardare ad un ampio spaccato della vicenda mafiosa.

Sulle molte carte, ora consultabili, dei numerosi procedimenti processuali avviati a margine della nota operazione Mori, e sull'importante scoperta della documentazione dell'Ispettorato generale di Sicurezza per la Sicilia, corpo costituito negli anni Trenta, è stata avviata, ormai alcuni anni fa, una ricerca che ha visto impegnato un gruppo di studiosi coordinato da Salvatore Lupo. Tale lavoro ha trovato una prima traduzione nel volume monografico della rivista "Meridiana", Mafia e fascismo (2008, n. 63), che ha fissato i termini per ripensare l'opera del regime nei confronti delle cosche siciliane. A partire da tale nucleo, ulteriori pubblicazioni hanno permesso di definire in termini ancora più precisi i differenti aspetti della questione.

Ad arricchire questo filone è, ultimo in ordine cronologico, il volume di Manoela Patti, che va a indagare la vicenda storica delle cosche dell'agro sud-orientale palermitano, da borgate come quelle di Santa Maria di Gesù o Ciaculli, ai comuni di Villabate e Bagheria. Lo studio può essere utilmente accostato al volume di Vittorio Coco, con il quale Patti dialoga fittamente a distanza, La mafia dei giardini (RomaBari, Laterza, 2013), che ha indagato l'altro versante dell'agro palermitano, quello nord-occidentale, e l'evoluzione delle sue differenti leadership criminali.

Fonte primaria è il materiale relativo al processo a quella che le indagini identificarono quale l'associazione a delinquere di Santa Maria di Gesù (comprendente in realtà l'intera l'area appena ricordata), che si sarebbe celebrato fra il 1930 e il 1932. Il 
volume, introdotto da una breve prefazione di Salvatore Lupo, è articolato su cinque capitoli in cui lo sguardo si muove fra i due poli di interesse: l'azione antimafia del regime e la realtà dell'underworld criminale che proprio tale opera permette di cogliere.

Nel primo capitolo viene richiamato il quadro generale di riferimento, ovvero la complessa penetrazione fascista in Sicilia che, a partire dalla metà degli anni Venti, produce l'operazione Mori. Con essa il tema dell'ordine pubblico è posto all'ordine del giorno, elevato a strumento in grado di giocare a favore del cambio degli equilibri politici, come già accaduto in passato. Sfuggendo a interpretazioni consolidatesi nel tempo, Patti evidenzia la complessità della strategia attuata con le retate del prefetto, definendola un'operazione politica volta a "guadagnare il consenso delle masse, recuperando quel rapporto diretto del centro con la periferia che l'intermediazione locale della politica aveva, per il fascismo, irreparabilmente compromesso" (p. 31).

Fra il secondo e il quarto capitolo l'attenzione si sposta, arrivando al cuore della ricerca. Attraverso un'attenta e paziente interpretazione delle fonti, Patti ricostruisce il fitto reticolato mafioso che, attraverso le carte della campagna repressiva, si riesce a intravedere. Con un focus che si sposta gradualmente da Villabate alle borgate più strettamente palermitane, i protagonisti della narrazione diventano quei numerosi gruppi di mafia, dai D’Agati ai Greco, passando dai Bontate, radicati già da alcuni decenni nell'area. Di questa realtà, Patti ci restituisce un quadro vivido e preciso, con cui è possibile seguire percorsi individuali e collettivi all'interno del network mafioso: i conflitti, i rapporti con gli altri gruppi sociali, l'acquisizione di posizioni avanzate nel mondo della produzione e del commercio, la costruzione di un ponte solido con la mafia statunitense, le strategie di alleanze coltivate tramite matrimoni e comparaggi, il controllo del territorio.

Questi gruppi sono fatti oggetto di un'indagine che si concretizza nel 1928 in una corposa retata, da cui scaturirà un "maxi- processo" ante litteram. È l'andamento processuale, tracciato nel quinto capitolo, a chiudere il cerchio rispetto alla riflessione avviata all'inizio del volume. L'esito giudiziario, al pari di molti casi coevi, è infatti deludente, fra assoluzioni o pene di scarso rilievo. Tale situazione produrrà un cambio di indirizzo nella strategia fascista: lo strumento processuale è accantonato a favore del più arbitrario ricorso al confino per i mafiosi, in un'azione repressiva, su cui la propaganda tace, ora condotta dall'Ispettorato. Sottolinea Patti che tale piega risponde all'obiettivo di costruire "uno stato pienamente fascista, privo di zone non fasciste o afasciste, e in cui la questione del consenso acquista carattere preminente" (p. 193).

Per quel che concerne il network criminale, invece, il tema centrale dell'intera narrazione è la continuità. Le cosche passate sotto la lente dell'autrice sono radicate prima dell'avvento del fascismo e sopravvivono a esso, protraendo la loro esistenza fino agli anni Ottanta, se non oltre, come dimostrano le indagini attentamente richiamate nel corso del testo. L'epilogo del volume, significativamente, ci porta nel convulso periodo che segue lo sbarco degli Alleati, dove ritroviamo questi gruppi nuovamente attivi dopo gli anni duri del confino. La mafia, ci ricorda Patti, non rinasce dalle sue ceneri dopo la repressione fascista, né viene rivitalizzata da oscuri complotti degli Alleati. L'azione del regime, anche se non indolore, non riuscì infatti a debellare il dato essenziale, ovvero la forza delle relazioni interne al sodalizio criminale, che si mantengono vitali, pronte ad essere reinvestite per negoziare un "ruolo all'interno del nuovo ordine costituito" (p. 219).

Diego Gavini

Isaia Sales, Storia dell'Italia mafiosa. Perché le mafie hanno avuto successo, Soveria Mannelli, Rubbettino, 2015, pp. 444, euro 19,50 .

L'autore - affermatosi nel tempo come osservatore dell'universo camorristico 
- ha esteso negli ultimi anni la sua prospettiva di studio andando a indagare in maniera più ampia l'insieme dei fenomeni mafiosi. In particolare, con Enzo Ciconte e Francesco Forgione, sta curando dal 2012 la pubblicazione dell'Atlante delle mafie (Rubbettino), con cui è stata avanzata in maniera pressante la necessità di studiare il "modello mafioso" nel suo complesso, ponendo il tema delle interconnessioni fra le reti criminali nel lungo periodo. Questa Storia dell'Italia mafiosa si pone in diretta continuità con tale approccio e rappresenta, al contempo, lo sforzo di condurre a una sintesi generale le osservazioni di Sales rispetto al tema della caratterizzante presenza di una criminalità di tipo mafioso in Italia.

A dispetto di quanto il titolo potrebbe lasciar supporre, il volume non è articolato in base a un criterio cronologico, ma secondo sette capitoli tematici (cui si aggiunge una corposa conclusione) che sviscerano gli aspetti ritenuti centrali da Sales per comprendere gli elementi cardine del modello mafioso e il rapporto che si instaura fra reti criminali, società e politica. In particolare, al vaglio di Sales vengono passate le origini di Cosa nostra, camorra e 'ndrangheta, gli elementi che le hanno differenziate nel tempo dal banditismo da un lato e dalla delinquenza comune dall'altro, il rapporto con la società meridionale e, infine, le modalità di inserimento nelle dinamiche economiche tramite canali legali e illegali. Categoria privilegiata che fa da filo conduttore all'intera ricostruzione è quella della "violenza di relazione", ovvero la capacità sviluppata nel tempo dagli attori dei gruppi di mafia di entrare in rapporto organico sia con gli strati sociali più deboli che con le classi dirigenti, tramite l'utilizzo della violenza privata quale canale di mediazione. Tale categoria è individuata come elemento chiave che sintetizza i motivi del successo di questo modello criminale e ne segna il discrimine essenziale rispetto ad altre forme delinquenziali.

La narrazione di Sales si appoggia, poi, sul tema del lungo periodo. L'origi- ne di queste consorterie è individuata già agli inizi dell'XIX secolo, in concomitanza con la fine del regime feudale nel regno borbonico, che sprigionerebbe il capitale di violenza privata precedentemente accumulato all'ombra di tale sistema; la definizione delle strutture organizzative sarebbe allo stesso tempo facilitata da rapporti trasversali, germogliati in particolare negli ambienti carcerari, che contribuiscono a diffondere pratiche comuni. Lo sviluppo decisivo avviene però, secondo Sales, con l'unificazione italiana; e anzi l'espansione del modello mafioso è associata al processo di nation building. Si colloca qui un altro tema forte della disamina, che indica nelle responsabilità delle classi dirigenti nazionali la chiave per comprendere la crescita mafiosa: prima per aver intessuto un'alleanza con l'aristocrazia latifondista meridionale, già profondamente legata alle forze della violenza mediatrice mafiosa, poi per aver persistito, nel periodo repubblicano, nel legame con queste ultime, al fine preservare gli assetti politici e sociali. A tale lettura si lega la prolungata critica a ogni rappresentazione della società del Mezzogiorno quale incubatrice dei fenomeni mafiosi per cause culturali o comportamentali.

In questa sua narrazione, Sales attraversa spesso terreni già battuti e riprende filoni interpretativi tradizionali, alcuni dei quali, peraltro, messi già in discussione dalla storiografia più attenta. Il punto di interesse centrale del volume è però nell'insistenza su due questioni che interrogano dall'esterno il mondo della ricerca e tentano un confronto con essa. Da un lato, infatti, Sales pone come punto dirimente la necessità di cambiare la prospettiva degli studi, concentrando l'attenzione non sulle singole organizzazioni criminali, bensì sull'indagine delle cause che hanno reso vincente il modello mafioso. Dall'altro, l'autore chiama direttamente in causa "la formazione degli storici italiani" (p. 20) per aver ridotto la questione mafiosa a un dato occasionale della vicenda nazionale. "La storia d'Italia, — scrive — leggen- 
do molti di questi testi, sembra a-mafiosa" (p. 20). Queste osservazioni non appaiono totalmente peregrine. Nel primo caso la storiografia è a lungo dominata da una specializzazione relativa alle singole consorterie di stampo mafioso, da cui solo a fatica si comincia a uscire nei più recenti sviluppi, tentando di rispondere alle domande che l'attualità inevitabilmente pone alla storia. Nel secondo caso, appare ugualmente opportuno il richiamo alla necessità di non relegare le dinamiche di formazione e sviluppo delle organizzazioni mafiose a fatto marginale della complessiva vicenda nazionale.

La prospettiva con cui Sales affronta le due questioni conduce, però, a quelli che sembrano essere i limiti più evidenti della ricostruzione generale, nel momento stesso in cui la narrazione appare quasi imbrigliata dalla necessità di dover corrispondere a queste gabbie interpretative. Rispetto al tema del modello mafioso, Sales sembra in parte evitare la riflessione su quegli aspetti di differenziazione che pur permangono fra i diversi aggregati mafiosi, specialmente se questo discorso viene declinato in una prospettiva di evoluzione storica. Non aiuta, in tal senso, la struttura del volume che sostanzialmente esclude la dimensione diacronica. Maggiori perplessità suscita poi il tema della presenza mafiosa nella storia politica e sociale italiana. Nel voler rimarcare questo dato, Sales appare infatti esasperare determinati caratteri, in particolare nel momento in cui eleva il rapporto mafia-politica quasi a paradigma totalizzante in grado di spiegare le dinamiche politiche nazionali, "da Crispi a Giolitti, da Andreotti fino a Berlusconi" (p. 17).

A margine di queste considerazioni, si può rimarcare il carattere positivo dello stimolo di volumi come questo, ma al contempo i vuoti che ancora attendono di essere colmati. In tal senso appare necessario che la ricerca scientifica risponda in maniera più sistematica a tali sollecitazioni, inserendosi nel dibattito con il rigore necessario.

Diego Gavini
Santa sede e sfide della modernità novecentesca

Giovanni Cavagnini, Per una più grande Italia. Il cardinale Pietro Maffi e la Prima guerra mondiale, Pisa, Pacini, 2015, pp. 206, euro 21.

Ricostruendo la vicenda del cardinale arcivescovo di Pisa Pietro Maffi, Giovanni Cavagnini ripercorre la storia unitaria dal punto di vista di uno dei suoi protagonisti: il clero cattolico. Il volume si concentra, infatti, sull'azione politica e pastorale di Maffi nella Grande guerra, ma la inserisce in una prospettiva di lungo periodo, che copre un lungo arco della storia dell'Italia unita.

Grazie a un attento esame delle fonti conservate nell'archivio arcivescovile e nella biblioteca del cardinale, aperte al pubblico nel novembre 2007, l'autore articola la sua ricerca in tre parti che rispondono a un criterio cronologico e tematico. Nella prima, che va dal 1858 al 1914, si chiariscono gli interessi scientifici ed intellettuali di Maffi, ovvero il suo rapporto con la modernità sorta dalla filosofia dei Lumi e dalla Rivoluzione francese, da cui i cattolici fecero discendere le cause ultime della Grande guerra. Ne emerge un profilo di grande interesse che intreccia passione per la scienza (grazie alla quale il giovane chierico divenne un astronomo di livello internazionale), impegno nell'organizzazione del movimento cattolico pisano all'interno di un disegno restauratore, acceso nazionalismo che in occasione della guerra di Libia lo spinse a bandire una crociata contro la "barbarie musulmana", sconfessata dalla Santa sede sulle pagine dell'“Osservatore romano".

La parte centrale della ricerca è però svolta nella seconda sezione, dedicata alla condotta assunta da Maffi nel corso del primo conflitto mondiale: dalla neutralità alla fase centrale della guerra e all'ultimo anno di scontri, inaugurato dalla crisi del 1917. Concentrandosi sulle relazioni dell'ar- 
civescovo con i superiori, le autorità politiche e i fedeli, Cavagnini ricostruisce le modulazioni nazionalistiche dei suoi interventi, mettendo in luce il costante tentativo di cristianizzarne la semantica. L'autore è interessato soprattutto a chiarire l'impatto di questa linea pastorale e politica sulla società civile in una fase della storia unitaria in cui la Questione romana era ancora aperta e la polemica liberale non mancava di sostenere l'inconciliabilità di fede e amor di patria. L'esame della ricchissima corrispondenza inedita, degli scritti pastorali e della fiorente stampa diocesana, completati dalle carte del ministero dell'Interno e dell'Archivio segreto vaticano, consente a Cavagnini di mettere in luce il ruolo costante di incitamento all'accettazione di qualsiasi sacrificio per il bene della nazione, svolto da Maffi nel corso del conflitto.

Le parole del cardinale, diffuse capillarmente attraverso la rete delle parrocchie, contribuirono a contenere l'ostilità nei riguardi dei governanti impedendone la trasformazione in rivolta generale. Pur trattandosi di una diocesi in cui la frequenza ai sacramenti e alle funzioni domenicali non era tra le più elevate in Italia, furono davvero pochi coloro che nel corso dei drammatici anni di guerra non ebbero bisogno del sostegno spirituale o materiale dei sacerdoti, pronti a farsi veicolo - come mostra questa ricerca — della linea del vescovo. In tal senso la ricostruzione qui presentata offre un contributo importante a comprendere il "mistero" del consenso o della resistenza alla guerra a fronte delle condizioni intollerabili in cui versavano amplissimi strati della società.

Nella terza e ultima parte Cavagnini affronta infine il tema della memoria in una duplice direzione: da un lato la promozione della memoria della guerra avanzata da Maffi nell'ultima fase del suo episcopato (1919-1931), dall'altra le commemorazioni postume relative all'arcivescovo pisano. Sul primo fronte la vicenda di Maffi coincide con quella di altri prelati italiani impegnati dapprima usare la memoria della guerra in una prospettiva di ricristianizza- zione della società, disposti poi a trovare nel nazionalismo uno dei terreni d'incontro con il fascismo. Nell'Italia della Guerra fredda il ruolo di Maffi nel primo conflitto mondiale e il suo contributo alla costruzione della "nazione cattolica" passò invece in secondo piano rispetto alla lotta antisocialista condotta dal cardinale prima e dopo la guerra.

Lucia Ceci

Giorgio Alessandrini, Federico Alessandrini. Santa sede tra nazismo e crisi spagnola (1933-1938), Introduzione di Renato Moro, Roma, Studium, 2015, pp. 366, euro 28.

Il volume raccoglie le schede di una lunga serie di articoli pubblicati tra la fine del 1933 e il 1938 da Federico Alessandrini (1905-1983), all'epoca giovane giornalista cattolico. I pezzi, firmati con diversi pseudonimi (Danubiano, Renano, Cid, Germanica, Verax, ecc.), sono dedicati alla vita internazionale e seguono, quasi giorno per giorno, il periodo che va dalla vittoria del nazismo in Germania alla vigilia della Seconda guerra mondiale.

Proveniente dal circolo romano della Fuci di Giovanni Battista Montini e Igino Righetti, Alessandrini era entrato nell'Ufficio stampa dell'Azione cattolica che, in seguito alla crisi del 1931, era stato trasferito in Vaticano, con il compito di redigere rassegne destinate non solo ai vertici dell'associazione, ma agli esponenti apicali della Segreteria di Stato e al segretario personale del papa, monsignor Carlo Confalonieri. Da quella posizione Alessandrini scrisse più di 350 articoli, materiale oggi difficile da reperire e di indubbio interesse. Si tratta di pezzi che passavano al vaglio attento del sostituto e che non potevano discostarsi dalla linea della Segreteria di Stato. Essi forniscono dunque al lettore informazioni importanti a diversi livelli. In primo luogo illuminano la figura di Federico Alessandrini, personalità significativa e ancora troppo poco nota nella storia del cattolice- 
simo italiano del Novecento. Basti pensare che dal 1946 al 1950 egli sarebbe stato direttore dell'organo dell'Azione cattolica "Il Quotidiano", nel 1961 vicedirettore dell'“Osservatore romano", dal 1970 al 1976 direttore della Sala stampa vaticana. Dunque, come sottolinea Renato Moro nell'introduzione al volume, "primo portavoce ufficiale laico della Santa sede" (p. 11).

Gli scritti qui raccolti offrono poi preziosi elementi sulla linea che la Segreteria di Stato intese seguire dinanzi alla crisi internazionale degli anni Trenta: essi recepivano infatti le indicazioni dei vertici vaticani che spesso intervenivano per correggerne il tiro, e danno di conseguenza bene il senso non solo del discorso pubblico costruito in anni cruciali dalla Santa sede (dato acquisito o acquisibile tramite altre fonti periodiche a essa più direttamente collegate come "L'Osservatore romano" e "La Civiltà cattolica"), ma dell'organizzazione in vigore al suo interno rispetto all'informazione da fornire ai cattolici italiani, al regime fascista, al governo del Reich, molto sospettoso verso i numerosi articoli sulla situazione tedesca firmati sulla stampa cattolica con un'ampia gamma di pseudonimi ora finalmente ricondotti alla penna dell'Alessandrini.

Altrettanto interessante è scoprire il materiale su cui lavorava Federico Alessandrini: stampa proveniente dal mondo cattolico francese con "La Documentation catholique", ma anche riviste schierate come "La Vie Intellectuelle" dei padri domenicani, "Esprit" di Emmanuel Mounier, periodici riconducibili a orizzonti laici con "La Revue des deux mondes", fogli marxisti con "L'International communiste", "Que faire?", giornali inglesi, tedeschi, russi, cui si aggiunge, naturalmente, la stampa italiana. Certo si trattava di articoli che Alessandrini leggeva per preparare la rassegna stampa destinata al segretario personale del papa e ai vertici della Segreteria di Stato, ma non erano in molti, nell'Italia di quegli anni, a poter disporre di uno sguardo così ampio sul mondo.

La prospettiva internazionale risalta anche nella grande varietà degli scenari pre- si in esame: dalla Francia alla Iugoslavia, dal Belgio alla Russia, dal Paraguay alla Cina, dal Messico agli Stati Uniti. A dominare questo quadro ricchissimo, tuttavia, è la situazione tedesca, rispetto alla quale si propongono letture tese a dimostrare l'assoluta incompatibilità tra ideologia nazista e dottrina cattolica, ma anche costanti informazioni sulle "persecuzioni" subite dai cattolici, mentre nulla emerge rispetto alle politiche antisemite. Meno attenzione, in proporzione, è dedicata al comunismo sovietico, la cui natura di feroce regime oppressivo è data praticamente per scontata, mentre molto pessimistico è il giudizio sulle democrazie che con la loro cecità laicista avevano finito, secondo Alessandrini, per accettare l'ammissione dell'Urss alla Società delle nazioni favorendo ulteriormente la diffusione mondiale dell'ideologia comunista e la politica dei Fronti popolari. Progressivamente viene meno anche la fiducia nei riguardi della tanto vagheggiata soluzione cattolica, autoritaria, cristiano-sociale e corporativa. Calpestata nell'Anschluss, abbandonata con l'Asse dall'Italia fascista, essa assume in Spagna caratteri e alleati che inducono Alessandrini a precisare che i cattolici non possono stare dalla parte di chi li combatte, ma non sposano l'ideologia della destra nazista, essi non si oppongono all'Asse, ma non accettano una "germanizzazione" del fascismo. Scartata la possibilità di una collocazione autonoma dei cattolici, a dominare è un senso di smarrimento. Non è forse un caso se gli ultimi articoli di Alessandrini risalgono al novembre 1938.

Lucia Ceci

\section{Fronti diversi della Grande guerra}

Paolo Ferrari, Alessandro MassignaNI, La guerra moderna. 1914-1918. Con documenti inediti, Milano, FrancoAngeli, 2014, pp. 288, euro 29.

Il volume proposto da Paolo Ferrari e Alessandro Massignani occupa un po- 
sto significativo nel panorama di pubblicazioni edite in occasione del centenario dell'entrata in guerra per più di una ragione. Il criterio adottato è stato, infatti, quello di proporre snelli testi introduttivi, seguiti da un'ampia selezione di fonti per lo più inedite. Dunque, non solo un testo di sintesi, ma il tentativo di far interagire la proposta di linee interpretative frutto di un complesso dibattito storiografico con la valorizzazione di uno spettro di documenti di varia natura.

Altra scelta condivisibile è stata quella di non tentare di esaurire il tema in tutte le sue declinazioni (dalla storia politica alle trasformazioni culturali e mentali, dal ruolo degli intellettuali nella propaganda fino alla "guerra dopo la guerra"). Un'impresa del genere avrebbe rischiato di liquidare parte di questi argomenti in termini sbrigativi e di sovrapporsi ai principali esiti della recente produzione, a partire dalla nota sintesi di Isnenghi e Rochat. I sette capitoli si soffermano invece su una serie coerente di problemi, mettendo al centro la stretta correlazione fra la storia militare, l'inedita rilevanza assunta dalla dimensione tecnologica e industriale, il binomio costituito da repressione e ricerca del consenso, come corollario della levée en masse.

La specificità saliente del volume sta proprio nell'attenzione dedicata alla storia militare, intesa ormai da decenni nel dibattito internazionale come un ricco campo di studi fondato sull'interazione fra una molteplicità di piani: gli aspetti tattici e strategici, il ruolo della guerra nella sperimentazione di nuovi apparati tecnologici, l'intreccio fra mobilitazione e riconversione dei sistemi produttivi, le trasformazioni dei quadri mentali innescate dalle esperienze belliche. La guerra dunque come grande acceleratore di trasformazioni, secondo una prospettiva che si attaglia perfettamente al primo conflitto mondiale e al suo ruolo di incubatore di dinamiche dispiegatesi compiutamente nei decenni successivi. Si tratta di una linea di ragionamento che gli autori sviluppano nel corso dei capitoli e che contribuisce a compensare la scarsa attenzione tradizionalmente riservata dai testi di lingua italiana a questo spettro di questioni, di cui Ferrari e Massignani sono specialisti riconosciuti.

Il tema della mobilitazione industriale e del nuovo ruolo dello Stato nell'economia è trattato in stretta connessione con l'immane pressione esercitata dalle esigenze del fronte sulla produzione; ad uno sguardo incrociato sulla situazione delle varie potenze belligeranti si affianca un'attenzione specifica al contesto italiano, al ruolo dell'industria militare nell'apparato produttivo e alla combinazione fra dirigismo e iniziativa privata che caratterizzò la penisola negli anni del conflitto.

$\mathrm{Al}$ caso italiano è dedicato un ampio spazio in tutte le sezioni; un altro pregio del volume sta tuttavia nell'aver calato le vicende nazionali nel più ampio quadro delle relazioni fra potenze e del concreto andamento del conflitto sui vari fronti.

Fra i documenti riprodotti è da evidenziare una serie di materiali inediti conservati nell'archivio dell'Ufficio storico dello Stato maggiore dell'esercito - un'autentica miniera di consultazione tutt'altro che facile - , nel Kriegsarchiv di Vienna e in altri archivi italiani ed esteri. Tali documenti permettono sia di approfondire un tema poco frequentato, ovvero la situazione del "nemico", con interessanti notazioni sulle condizioni dell'esercito austroungarico nonché sulla percezione che i suoi comandi avevano della compagine italiana, sia di affrontare una serie di questioni chiave, quali il nuovo peso dell'aeronautica nella ricognizione, ma anche in azioni offensive, al di là del mito dei grandi aviatori a cui è legata nella cultura di massa la memoria della Prima guerra mondiale.

Fra le fonti a stampa presentate nel volume si segnalano i materiali ufficiali prodotti dal Comitato centrale per la mobilitazione industriale del ministero della Guerra, le coeve analisi quantitative elaborate da Riccardo Bachi, stralci dalle memorie dell'industriale Ettore Conti, scritti politici come quelli di Timeus (Ruggero 
Fauro), fra i pionieri del nazionalismo antislavo.

Il lavoro di Ferrari e Massignani conferma in definitiva l'efficacia di un confronto diretto con le fonti, in grado di sprigionare una molteplicità di suggestioni e angolature purché frutto di un attento lavoro di selezione critica; il taglio e il criterio compositivo del volume lo rendono fra l'altro particolarmente adatto quale testo di approfondimento in ambito scolastico e universitario.

Francesca Cavarocchi

Antonio VArsori, Radioso Maggio. Come l'Italia entrò in guerra, Bologna, il Mulino, 2015, pp. 215, euro 15.

Uscito in coincidenza con l'anniversario della dichiarazione di guerra del 24 maggio 1915, questo volume di Antonio Varsori, noto studioso di storia delle relazioni internazionali, contribuisce al profluvio di pubblicazioni uscite in occasione del centenario della Grande guerra con il proposito di analizzare da vicino proprio l'azione di figure ed eventi che determinarono l'ingresso italiano nel conflitto europeo. In coerenza con la formazione dell'autore, il testo presenta un approccio agli eventi di taglio fortemente istituzionale, privilegiando naturalmente la storia diplomatica, considerata però qui nei suoi profondi intrecci con quella politico-parlamentare. Proprio lo stretto nesso e la forte interazione fra le due dimensioni è, infatti, uno degli aspetti distintivi di questo lavoro, specialmente se si considera, stando alle parole dell'introduzione, che nel considerare la vicenda dell'intervento italiano "fattori domestici e fattori internazionali" "sono stati presi spesso in esame separatamente" piuttosto che nella loro intima correlazione (p. 8).

Approcci non considerati, come quelli riconducibili alla storia sociale, economica e culturale (anche se da questo ultimo punto di vista non mancano significative concessioni al ruolo mobilitante svolto in relazione agli eventi dal discorso di retori come D'Annunzio), sembrano ritenuti del resto da Varsori secondari, quanto meno nel caso specifico della scelta interventista italiana.

La lettura del volume non sfugge così all'impressione di fondo che il taglio prescelto per ragioni di appartenenza disciplinare risulti allo stesso tempo nella convinzione dell'autore anche il più adatto alla messa a fuoco degli eventi in questione, vista la loro stretta dipendenza da fattori come il protagonismo politico di poche personalità, fra cui primeggiano Salandra e Sonnino, e dato soprattutto il carattere assolutamente decisivo dei fatti accaduti nel breve arco di tempo rappresentato dal cosiddetto radioso maggio intercorso tra la firma del Patto di Londra e l'entrata in guerra.

Pur a fronte di sommovimenti storici profondi in azione negli anni che precedono l'avvento della guerra, per Varsori non si può prescindere dalla specifica congiuntura rappresentata da quelle poche settimane; una congiuntura dominata da trattative segrete, coincidenze e calcoli politici di cui il libro, muovendosi fra punto di vista interno e politica internazionale, ha appunto l'ambizione di fornire un racconto puntuale.

Del resto, se non sono mancati in passato contributi attenti agli stessi aspetti politici e diplomatici, da quelli di Brunello Vigezzi (Da Giolitti a Salandra, Firenze, Vallecchi, 1969; L'Italia neutrale, Milano, Ricciardi, 1966) fino al più recente di Gian Enrico Rusconi (L'azzardo del 1915, Bologna, il Mulino, 2009), mancava un dettagliato approfondimento non tanto del periodo della neutralità, su cui si concentra ad esempio il citato Rusconi, quanto soprattutto del mese precedente l'avvio del conflitto.

In realtà, a dispetto di questi dichiarati propositi, la prima metà del testo si compone di due capitoli di inquadramento generale più utili forse a un lettore non specialistico; è infatti solo nei due successivi capitoli dei quattro complessivi che si en- 
tra nel vivo dell'argomento trattato e l'ampio ricorso a fonti diplomatiche, integrate da quelle diaristiche ed epistolari di alcuni dei principali interpreti degli eventi come Sonnino o Giolitti, consente una cronaca serrata e quasi quotidiana degli accadimenti. In quel maggio, ricorda opportunamente Varsori, finirono peraltro per intrecciarsi e rispecchiarsi continuità e discontinuità della storia italiana; se classici topos sull'inaffidabilità della politica estera italiana sembravano essere confermati dalla doppiezza che accompagnava talune fondamentali decisioni, da parte di chi manteneva in piedi più negoziati, molte delle scelte opportunistiche sottese a tali manovre avevano secondo l'autore proprio l'obiettivo di uscire da quello stigma infamante e garantire invece un futuro status di rispettabile potenza all'Italia. L'azione di molti interventisti, così come la loro retorica, è presentata da Varsori come l'espressione di una volontà di rottura nella vicenda dello Stato liberale, di cui gli stessi interventisti volevano superare quelle che a loro parevano ambiguità e debolezze, tanto che lo stesso Mussolini farà del "maggio radioso" uno spartiacque epocale nella storia del paese.

Il fantasma ingombrante del fascismo aleggia anche nell'ultimo capitolo nel quale si affronta un nodo interpretativo particolarmente dibattuto con l'avvento della Repubblica, ossia se il "maggio radioso" abbia o meno rappresentato un "colpo di Stato", una sorta di anticipo della presa del potere mussoliniana. Pur non pronunciandosi in termini netti, Varsori pare escludere una tale eventualità, anche alla luce del giudizio offerto sul comportamento tenuto dalla monarchia. Nelle sue pagine l'opera svolta dal re sembra, infatti, secondaria e non imputabile di particolari forzature; più succube degli eventi che suo protagonista, il Vittorio Emanuele III di Varsori sembra assoggettarsi al dinamismo di Salandra e Sonnino, determinati nel perseguire un disegno che, fallito sul medio periodo, mirava a collegare intimamente decisioni, ed eventuali successi in politica estera a considerazioni di politica interna di segno conservatore e antigiolittiano.

Se la ricostruzione complessiva mette ben in luce come nel campo di studio privilegiato da queste pagine, ossia i vertici della politica italiana e le sue sedi istituzionali, l'opzione dell'entrata in guerra risultasse tutt'altro che condivisa, lasciano qualche dubbio invece alcuni giudizi sull'atteggiamento del resto della nazione, con allusione evidente alle sue classi subalterne. Sulla completa apatia rispetto alle grandi vicende in corso di un'Italia ancora largamente contadina, scettica ma sostanzialmente indifferente all'ipotesi di un intervento bellico, le ricerche sul campo non sempre paiono confermare un tale quadro. Recenti studi (come quelli raccolti di recente in Abbasso la guerra! Neutralisti in piazza alla vigilia della Prima guerra mondiale in Italia, a cura di F. Cammarano, Milano, Le Monnier, 2015) mostrano al contrario un Paese che vive per quasi un anno in una sorta di fibrillazione continua. Infine, la stessa dinamica di piazza, diversamente da quanto affermato nel volume, appare riconducibile al solo coinvolgimento di D'Annunzio, mostrandosi ben viva in tante città italiane già nei mesi precedenti all'episodio di Quarto, a testimoniare una volta di più la difficoltà di concentrare la dimensione della partecipazione popolare sulla discussione in atto alle sole giornate del "maggio radioso".

Marco Manfredi

Giuseppe Ferraro (a cura di), Dalle trincee alle retrovie. I molti fronti della Grande guerra, Arcavacata di Rende, Icsaic, 2015.

"Figli di un dio minore": potrebbe essere questo il sottotitolo del volume sul primo conflitto mondiale pubblicato dall'Istituto calabrese per la storia dell'antifascismo e dell'Italia contemporanea con il contributo di una dozzina d'autori, suddivisi in tre diverse sezioni. Di esse solo quella centrale è esplicitamente dedicata 
alla Calabria, mentre i vari saggi utilizzano, qualcuno più, altri meno, fonti di diverso genere, non esclusi i materiali d'archivio. "Figli di un dio minore", quindi, non certo per lo scarso interesse dei temi affrontati, ma per la scelta di percorsi meno battuti e scontati, e proprio per questo maggiormente interessanti, di quelli indagati recentemente dalla storiografia della Grande guerra. Nell'accavallarsi di pubblicazioni e ricerche sulle vicende e gli aspetti del primo conflitto mondiale, che il centenario lasciava supporre, ma non prevedere in questa mole, il testo si segnala per l'originalità dei suoi approcci. Già il contributo che inaugura la prima sezione, per esempio, si occupa del dibattito sulla carta stampata in lingua italiana in Brasile (J.F. Bertonha), che mette a confronto, tramite appunto un'analisi dei principali "fogli", due delle comunità più importanti dell'emigrazione italiana in Sud America, quella "italiana" appunto e quella "asburgica", attraverso la componente sud-tirolese. Non meno singolari sono i percorsi con cui si esplora la genesi del sindacalismo nazionale nei suoi rapporti con l'adesione al conflitto da parte italiana (G. Sacchetti). O l'approccio iniziale alla scuola sud-tirolese, all'indomani della conquista (A. Dessardo), da parte delle autorità scolastiche italiane, in una prospettiva di fattivo confronto, che il fascismo avrebbe poi stroncato. La seconda sezione è dedicata specificamente al caso calabrese. La serie di temi regionali che vi vengono sviluppati (G. Ferraro, L. Intrieri, V.A. Tucci, T. Grano, R. Liberti, G. Marcianò) non hanno peraltro nulla di "localistico" e tanto meno di asfittico, dando piuttosto il la a una serie di riflessioni sul nodo dei rapporti tra regioni della Penisola e carattere nazionale del nostro Paese. La terza si confronta con aree di indagine forse più note, o comunque esplorate dalla storiografia, quali la costruzione della memoria e l'elaborazione del lutto a livello anche di microrealtà sociali o il rapporto storia-cultura $(\mathrm{M}$. Gualtieri, S. Inglese, M.T. Sorrenti, F. Corigliano), attraverso le vicende di un singo- lo intellettuale, dello spessore peraltro di Corrado Alvaro.

Non tutti gli interventi risultano dello stesso livello e non mancano alcune ingenuità, relative soprattutto alle operazioni militari, che una revisione più accurata del testo avrebbe potuto evitare. D'altro canto si tratta di un nodo storiografico reputato sempre fin troppo noto, anche se in realtà, alla prova dei fatti, appare spesso non adeguatamente conosciuto. Senza nulla togliere agli stimoli presenti negli altri interventi (per esempio, sul ruolo svolto dai cattolici e dal Vaticano allo scoppio e nel corso del conflitto), meritano certamente una segnalazione le analisi relative alle scritture di guerra calabresi (G. Ferraro), nonché il saggio (T. Grano) relativo all'apporto, intellettuale e militare, di un giornalista come Roberto Taverniti, che trovò la morte sul Carso. Quest'ultimo offre, infatti, la possibilità da un lato di ripensare a un rapporto, quello del giornalismo col conflitto, spesso limitato ai nomi, pur di assoluto rilievo di Albertini, Mussolini, Barzini, Fraccaroli o Alessi, dall'altro di seguire il percorso con cui l'interventismo democratico affrontava il conflitto nella prospettiva della partecipazione delle classi popolari, molto prima dell'esperienza dei reduci e del "reducismo" (Lussu, Bellieni, Mameli, Bergamo, ecc.). Il contributo di Ferraro ripropone invece la problematica, attraverso il caso calabrese, della partecipazione del Meridione alla guerra. Una partecipazione oltretutto che, nota e spesso ribadita per i casi resi celebri dalla cronaca (Brigate "Salerno, "Catanzaro", "Aosta"), meriterebbe in realtà un approfondimento maggiore. La sola consistenza dell'apporto numerico dato soprattutto alla fanteria da paesi dell'Italia meridionale, che scontavano ancora gli strascichi, anche sanguinosi, della lotta al brigantaggio, è sempre in attesa infatti di un'indagine specifica. In realtà proprio la tematica dell'adesione e/o del rifiuto del conflitto, nella forma più spontanea della renitenza o della diserzione, e dei conseguenti procedimenti penali, da parte delle regioni 
meridionali sarebbe in grado di apportare alla storiografia della guerra un contributo quanto mai significativo.

L'ultima sezione affronta l'esame del difficile rapporto di Aby Warburg con l'Italia e la sua decisione di prendere parte alla guerra contro il "mondo tedesco", se non - almeno inizialmente — contro la Germania; la costruzione "monumentale" della memoria e la conseguente elaborazione del lutto e del suo carattere nazionale da parte di comunità locali, spesso poco o male integrate nella compagine statale; l'evoluzione, infine, del rapportarsi al conflitto di un intellettuale come Corrado Alvaro, ripercorsa attraverso lo sviluppo e la conclusione della sua parabola poetica. In un certo senso essa viene a costituire la sintesi delle prime due, o quanto meno finisce col riannodarne in modo diverso i fili conduttori. Riporta, infatti, le emergenze squisitamente locali, quanto meno nelle realizzazioni monumentali, segnate spesso dalle polemiche tra la produzione "seriale" del ricordo dei caduti e il suo "ripensamento" da parte di personalità di caratura maggiore, a una dimensione più vasta, articolata e naturalmente complessa. Inversamente - ed è forse l'apporto più interessante che si ricava dall'esame del "caso" Warburg - solo da una prospettiva transnazionale, sia pur invischiata e gravata dall'adesione nazionalistica alla causa tedesca, si riesce a comprendere come anche il più locale dei dibattiti o delle manifestazioni (sia pure a opera di studenti liceali di una città ancora segnata dal terremoto, come Catanzaro) si inserisca già in un contesto "globalizzato", anche, se non soprattutto, sotto il profilo culturale. L'indicazione che ne consegue è preziosa, perché non è affatto scontata e non sempre viene seguita in quelle indagini storiografiche "a km zero", per usare un'espressione di Mario Isnenghi, che vengono condotte e che il centenario ha incentivato soprattutto ma non solo, nel Nord-est della Penisola.

Gli abstract degli interventi e i profili dei diversi autori, che vengono acclusi in appendice, sono lodevolmente presenti an- che in lingua inglese. Si avverte peraltro la mancanza di un indice dei nomi, che in un'opera che presenta spiccatamente le caratteristiche tipiche degli atti congressuali avrebbe senza dubbio facilitato la fruizione scientifica.

Paolo Pozzato

Aspetti di storia del fascismo: organizzare il tempo libero, costruire l'impero

Valeria Deplano, L'Africa in casa. Propaganda e cultura coloniale nell'Italia fascista, Firenze, Le Monnier, 2015, pp. VI202, ill., euro 15.

Fino a non molti anni fa il colonialismo è stato trattato, nell'ambito degli studi storici, come un corollario della storia d'Italia "distinto da essa al punto da poter essere eliminato dalla ricostruzione storica o, al limite, studiato separatamente". In altre parole "all'esperienza coloniale veniva negato qualunque tipo di impatto reale sulla nazione".

È solo dall'inizio degli anni Ottanta del Novecento e, un decennio dopo, "sulla spinta degli studi culturali" che vari studiosi hanno iniziato a riflettere "sulle connessioni tra colonialismo italiano e storia nazionale, integrando il fronte interno nell'analisi del fenomeno espansionista".

In particolare lo studio delle immagini e dell'immaginario ha rivelato come le rappresentazioni coloniali "suggerissero anche specifici rapporti di genere, specifici concetti di normalità e difformità, di superiorità e inferiorità, che condizionavano profondamente l'intera visione del mondo degli italiani".

Al centro della ricerca di Valeria Deplano vi sono gli anni della dittatura fascista, ma non solo quelli. Il saggio, infatti, ha il merito di rendere evidente come il fascismo seppe rielaborare radicalmente le categorie dell'imperialismo coloniale, intervenen- 
do su un terreno che era di per sé già stato reso molto fertile nei decenni che avevano preceduto l'avvento della dittatura.

Negli anni Venti e Trenta la cultura coloniale fu, come sostiene l'autrice, "il terreno d'incontro di due aspetti cruciali del programma di Mussolini: la riproposizione, prima nelle intenzioni e dopo nei fatti, di una politica di potenza che riservava all'espansione coloniale un posto di spicco nell'agenda della politica estera: e la volontà di mobilitare attorno alle parole d'ordine del fascismo il popolo italiano nella sua interezza, e di plasmare, sulla base di queste, un "uomo nuovo"'.

Nel processo di costruzione di uno stato totalitario, che "presupponeva da un lato il controllo governativo su tutte le istituzioni, anche quelle culturali, e dall'altro la mobilitazione della nazione attorno ai cosiddetti valori fascisti”, il terreno prescelto dal fascismo per il primo decisivo cimento è la Libia: "una colonia appena riconquistata, che il regime voleva trasformare in un nuovo nucleo di italianità".

Il tema della coscienza coloniale da creare e diffondere tra gli italiani sarà la palestra ideale per mantenere vivo lo spirito guerriero della nazione e rendere accettabile agli italiani la volontà di lavorare, da parte del fascismo, a una politica di potenza basata sull'aggressività militare. I difficili e precari equilibri di pace generati dalla cessazione delle operazioni belliche della Prima guerra mondiale avevano lasciato presupporre, per il prossimo futuro dell'Europa e del vecchio mondo, una probabile ripresa del conflitto. E questa prospettiva, col passare degli anni, veniva via via concretizzandosi nella dualità che contrapponeva, da un lato, l'affermarsi del fascismo in Italia, in Germania e in altre nazioni europee ed il mancato "disarmo" dei nazionalismi; dall'altro, la minacciosa constatazione per i politici dei Paesi di tradizione democratica che una idea, in grado di mandare in frantumi gli antichi assetti di egemonia classista e di potere capitalistico, era stata capace, per la prima volta nella storia, di farsi Stato.
Accanto a questo contesto internazionale nell'esperienza dell'organizzazione dello stato fascista in Italia c'è, ricorda Valeria Deplano, "un istituto completamente rinnovato cui era affidata una precisa missione politica e culturale: quella di creare una 'coscienza coloniale' tra gli italiani".

Il saggio focalizza il proprio interesse sugli effetti centripeti dell'espansionismo e prende in esame le attività degli istituti geografici e coloniali per studiare i meccanismi di diffusione della coscienza coloniale, analizzandone la storia, ma più in generale il loro divenire "una parte della storia dell'Italia, e della formazione degli italiani come comunità nazionale".

La storia degli istituti colonialisti durante il fascismo è quindi ora "la storia dei progetti del regime in campo coloniale, delle loro intersezioni con il progetto di irreggimentazione culturale, e della loro concretizzazione in iniziative propagandistiche e culturali destinate all'educazione della nazione".

La prima parte del saggio si sofferma dunque sulle motivazioni dell" "urgenza coloniale del governo", inserendola nel contesto europeo e prefascista. A partire dall'anno della svolta, nel 1926, allorché Mussolini inaugura la nuova politica culturale che doveva portare la coscienza coloniale a diventare parte della coscienza nazionale, la ricerca si preoccupa di sviscerare la vicenda della rapida costruzione delle fabbriche del consenso coloniale fino alla apparente svolta rappresentata dall'aggressione all'Etiopia nel 1935 e alla successiva proclamazione dell'impero.

Da quel momento, sottolinea l'autrice, la scuola, l'università, le strutture propagandistiche si occupano di preparare gli italiani "a comportarsi come un popolo imperiale: protagonista del proprio destino, razzisticamente superiore, proiettato verso un mondo non più costretto dai confini angusti della penisola".

Il saggio si occupa infine di raccontare quanto accade dopo il 1936 sino alla Seconda guerra mondiale, per arrivare poi — assecondandone i tratti di continuità 
- fino ai primissimi anni della Repubblica. "Si tratta - sottolinea Deplano - di un punto di partenza necessario per comprendere i rapporti tra fascismo e identità nazionale durante il Ventennio, ma anche per iniziare a pensare al colonialismo non come parentesi, bensì come processo culturale con radici salde, da cui è necessario indagare le permanenze al di là della perdita dell'impero e dei cambiamenti istituzionali".

Adolfo Mignemi

Robert Mallett, Mussolini in Ethiopia, The Origins of Fascist Italy's African War, 1919-1933, New York, Cambridge University Press, 2015, pp. VII-228, euro 87.

Gli anniversari sono sempre occasione di riflessione storiografica, come dimostra il centenario della Grande guerra. Nel 2015 scadevano anche gli ottant'anni della Guerra d'Etiopia (1935-36) e gli studiosi hanno voluto approfittarne per aggiornare il quadro delle ricerche, estendendo la durata del conflitto fino alla caduta dell'impero fascista, come ha fatto Nicola Labanca (La guerra d'Etiopia 1935-1941, Bologna, il Mulino, 2015), oppure ripercorrendone le origini, come ha fatto Robert Mallett con questo suo Mussolini in Ethiopia.

Mallett è un ex ricercatore che ha continuato l'attività di studioso indipendente. È conosciuto per i suoi studi sulla Regia marina nel periodo 1935-1940 e la politica estera mussoliniana degli anni Trenta, ma il suo libro sull'Etiopia mira a delineare le origini e la preparazione diplomaticomilitare del conflitto dalla Grande guerra fino al 1935.

I primi capitoli (pp. 1-32) sono dedicati alle origini della guerra rintracciate nelle ambizioni nazionaliste frustrate dalla "vittoria mutilata" e nell'ascesa del fascismo, postosi come attore desideroso di rovesciare l'ordine internazionale postbellico, per dare all'Italia un proprio "spazio vitale", definito secondo precisi contorni geopolitici, nel Mediterraneo e in Africa. Mallett mette in evidenza l'importanza attribuita alla possibile alleanza con la Germania, il cui potenziale economico e militare era centrale per i progetti del duce (p. 22).

Nella parte centrale del volume (pp. 52123) è evidenziato come la crisi economica del 1929 e l'ascesa del nazismo indussero il duce a ritenere che fosse arrivato il momento per l'Italia di espandersi, eventualmente ai danni della Iugoslavia. Tuttavia, l'opposizione dei capi militari, consci del disastro nel caso di una guerra contro l'alleanza franco-iugoslava, spostò l'attenzione strategica del dittatore sull'Africa orientale (p. 63). A partire dalla metà del 1932, iniziò la preparazione militare all'invasione dell'Etiopia, un obiettivo che offriva all'Italia fascista la possibilità di vendicare la sconfitta di Adua e di diventare una "grande potenza africana" (p. 66).

Da questo punto, Mallett si muove in una doppia prospettiva analizzando contemporaneamente la preparazione militare alla guerra e i problemi diplomatici che indussero Mussolini a fermare, per il momento, le velleità espansioniste di Hitler. In seguito al tentativo di colpo di stato nazista in Austria dell'estate 1934, il duce ritenne che una riduzione delle truppe in Europa avrebbe potuto danneggiare l'Italia (p. 97) e perciò, per tenere in scacco temporaneamente Hitler e realizzare i propri progetti africani, decise di allearsi con la nazione che lui e i fascisti detestavano maggiormente (p. 100): ebbe così inizio un riavvicinamento temporaneo tra Francia e Italia, culminato nell'incontro Laval-Mussolini del gennaio 1935.

Nell'ultima parte del volume (pp. 124217), procede la narrazione parallela dei preparativi militari, soprattutto delle difficoltà logistico-organizzative e dei contrasti tra Badoglio e De Bono, e diplomatici, facendo entrare in gioco prepotentemente l'ultimo attore chiave: la Gran Bretagna. Anche in questo caso Mallett tiene a sottolineare quello che presenta come un tatticismo di Mussolini che, sapendo dell'apprensione che l'espansione italiana in Etiopia poteva causare a Londra, da un 
lato negò l'esistenza di preparativi in Africa Orientale e dall'altro utilizzò il timore del riarmo tedesco per cercare il consenso britannico all'invasione dell'Etiopia (p. 147).

La difesa dell'ordine societario da parte britannica e il fatto che Mussolini non avrebbe mai accettato una soluzione che non avesse corrisposto al totale dominio sul paese africano (p. 200) portarono al rapido inasprirsi della tensione tra le due potenze. Il tutto culminò nella mobilitazione delle flotte britannica e italiana dell'estate 1935 che favorì nel contempo anche la distensione dei rapporti italo-tedeschi. Nonostante la minaccia britannica, Mussolini era deciso a conquistare il paese africano, ritenendolo un diritto per l'Italia (p. 210): perciò neutralizzò i tentativi di mediazione britannici e in questo modo, mettendo in crisi il sistema di sicurezza collettivo della Società delle nazioni e aprendo la strada a quella "war of revenge" (p. 218) che secondo Mallett fu la Guerra d'Etiopia.

Il saggio è scorrevole e in alcune parti basato su ricerche negli archivi del ministero degli Affari esteri e dell'Esercito, oltre che all'Archivio centrale dello Stato, mentre l'apparato delle note a volte è molto avaro. In quarta di copertina, com'è consueto nell'editoria anglosassone, è possibile leggere giudizi positivi di storici come James Burgwyn e del recentemente scomparso Christopher Duggan, ma nel giudicarlo forse occorre tenere conto della diversità di prospettive tra la storiografia italiana e quella anglosassone.

Per un lettore britannico il volume infatti offre un'efficace sintesi delle questioni diplomatico-militari connesse alla guerra. Il testo però manca all'inizio di un chiaro posizionamento storiografico, un aspetto che si riflette anche nel ridotto numero di autori italiani citati da Mallett. La politica coloniale dell'Italia liberale è trascurata, privando Mallett della possibilità di ragionare sul nesso continuità/discontinuità con quella del regime. Più in generale, a un lettore italiano risalta che le tesi di
Mallett riguardanti l'espansione coloniale fascista riprendono quanto già presente negli studi di Angelo Del Boca e Nicola Labanca. Per quanto riguarda la preparazione militare dell'invasione, Mallett non aggiunge molto a quanto illustrato a suo tempo da Giorgio Rochat (Militari e politici nella preparazione della campagna d'Etiopia. Studio e documenti 1932-1936, Milano, Angeli, 1971). Un aspetto più originale è l'analisi della tensione diplomatico-navale nel Mediterraneo, che però lo stesso Mallett aveva già tracciato in precedenza (The Italian Navy and Fascist Expansionism 1935-1940, Londra, F. Cass, 1998). Infine, l'analisi del rapporto con la Germania è arricchito con nuove fonti, ma rientra nelle prospettive elaborate da Jens Petersen, Enzo Collotti e MacGregor Knox. D'altra parte, la mancata traduzione in inglese delle opere degli studiosi italiani che abbiamo citato, probabilmente rende il volume di Mallett importante se osservato dalla prospettiva dei paesi anglosassoni, dove un saggio che discutesse complessivamente il problema delle origini della Guerra d'Etiopia in effetti mancava.

Fabio De Ninno

Elena Vigilante, L'Opera nazionale dopolavoro. Tempo libero dei lavoratori, assistenza e regime fascista 1925 1943, Prefazione di Guido Melis, Bologna, il Mulino, 2014, pp. 217, euro 20.

Com'era organizzato il tempo libero dei lavoratori dal regima fascista? È questa la domanda a cui il documentato libro di Vigilante prova a dare una risposta affrontando lo studio dell'istituzione preposta alla sua promozione: l'Opera nazionale dopolavoro. In poco più di duecento pagine se ne ripercorrono le diverse fasi: dalla sua istituzione, nel 1925, su richiesta degli ambienti sindacali fascisti che miravano a una maggiore penetrazione nella società attraverso la "rieducazione delle masse", al successivo e lungo decennio di commis- 
sariamento, originato da un assetto istituzionale dagli equilibri sostanzialmente precari; dalla breve fase di risistemazione degli organi direttivi, a partire dalla quale, nel 1937, l'Ond avrebbe dovuto operare su un piano più propriamente politico, fino a tutto il successivo periodo di guerra, quando l'ente fu a più riprese sottoposto nuovamente a commissariamento. Il focus sul caso della Basilicata, su cui l'autrice si sofferma nell'ultima parte del libro per sottoporre a verifica l'azione dell'Ond nelle aree rurali, impreziosisce il quadro.

Un lavoro di ricostruzione tutt'altro che semplice, anche per l'assenza dell'archivio dell'Ond che, andato disperso nel dopoguerra, ha impegnato Vigilante in una scrupolosa e accurata ricerca delle sue carte negli archivi delle diverse istituzioni fasciste e nello studio di una vasta letteratura grigia dell'epoca. I risultati sono significativi e si collocano all'interno di quell'orizzonte tematico e interpretativo che più ha caratterizzato la ricerca storiografica sul fascismo dell'ultimo ventennio.

Acute valutazioni e specifici studi sull'Ond non sono certamente mancate in passato. Com'è noto, la novità rappresentata da questa istituzione era già stata colta nel suo divenire da Palmiro Togliatti, che in una delle sue lezioni sul fascismo tenute a Mosca agli inizi del 1935 non solo aveva visto nell'Ond una delle misure adottate dal regime per arrivare all'organizzazione dello Stato corporativo, ma ne aveva riconosciuto un'efficacia specifica tra le organizzazioni del fascismo per la sua capacità di soddisfare alcuni dei bisogni reali delle masse. Una lettura sostanzialmente confermata da Renzo De Felice nei suoi studi sugli anni del consenso al fascismo e che trova una prima articolata sistematizzazione agli inizi degli anni Ottanta con il volume di Victoria De Grazia, che ebbe il merito di far emergere le specificità dell'ente in tutta la loro complessità, sia come organizzazione del consenso che come istituto volto all'ampliamento dei consumi di massa.

Il libro di Vigilante non si discosta da questo quadro interpretativo, ma vi appro- da da un diverso angolo visuale: quello dell'organizzazione e della distribuzione del potere all'interno dell'Ond, attraverso l'esame delle norme governative, delle direttive del Pnf e dei resoconti sulla crescita del dopolavoro stesso. Di conseguenza, sono le tensioni e i conflitti - nemmeno tanto sotterranei - tra l'ispirazione politica dell'ente e la vocazione accentratrice dello Stato, ai cui apparati era stato affidato il controllo, a imporsi nell'analisi dell'autrice, costituendo un aspetto di particolare interesse del volume. Conflitti e tensioni che, percorrendo sostanzialmente tutta l'esperienza del regime fascista, come rileva Melis nella Prefazione, contribuiscono ulteriormente alla definizione dell'immagine di un fascismo tutt'altro che monolitico.

In questo quadro, le valutazioni sugli aspetti di novità dell'ente vengono spesso filtrate sia dal confronto con il precedente periodo liberale, di cui si evidenziano continuità e rotture, sia da quello con i coevi dibattiti e modelli europei e americani, rispetto ai quali si provano a cogliere specificità e affinità. Non a caso l'autrice inserisce l'istituzione dell'Ond nel processo di modernizzazione della società italiana fra le due guerre e, al contempo, ne legge l'impatto su di essa come indice del cambiamento avvenuto con la prima guerra mondiale — in Italia, ma non solo nel rapporto fra Stato e cittadino. Come tutta una serie di enti nati o rinnovatisi a partire dalla metà degli anni venti e operanti nel campo delle politiche sociali, l'Ond è senz'altro emblematica dell'assorbimento nell'ambito dello Stato di prerogative e funzioni precedentemente considerate estranee ai suoi apparati. Tuttavia, Vigilante non esita a precisare che in ambito dopolavoristico l'Opera "non inventò quasi nulla, limitandosi ad assorbire, per contenuti e modalità, le variegate attività promosse dalle forme disparate di associazionismo presenti nell'Italia liberale" (p. 38). Allo stesso modo si rileva come il problema dell'organizzazione del tempo libero dei lavoratori era stato in realtà posto 
in Italia già nell'immediato dopoguerra, frutto di una cultura tayloristica penetrata a diversi livelli in sede internazionale. Illuminanti, in proposito, sono le raccomandazioni dell'Organizzazione internazionale del lavoro. Rispetto a queste valutazioni, altra cosa è invece la straordinaria capacità che il fascismo ebbe di adattare la soluzione del problema al proprio progetto autoritario di governo delle masse, finalizzandolo alla costruzione del consenso.

Nel disegno del gruppo dirigente dell'Ond vi era l'obiettivo di fare del dopolavoro lo strumento per il "miglioramento materiale e morale dei lavoratori indispensabile per il progresso della nazione" - così recitava l'opuscolo illustrativo delle attività — in modo da favorire la diffusione del sentimento patriottico e sottrarre i lavoratori alle "nefaste influenze" delle ideologie sovversive del passato (p. 32). Il progetto avrebbe dovuto garantire la piena fascistizzazione dei lavoratori, controllandone il tempo libero anche al di fuori e dopo l'orario di lavoro. Non stupisce che nel 1937 il dopolavoro sia arrivato a contare 3.180.000 tesserati (il costo della tessera, 5 lire, era ammortizzato dalla possibilità di acquistare generi di prima necessità a prezzi calmierati).

La funzione assistenziale del dopolavoro giocò un ruolo importante nel perseguimento di questi obiettivi e il Pnf, nella sua funzione di "grande pedagogo" degli italiani pronto ad occupare tutti i possibili spazi della società, ne fu il principale artefice. Su questo, come Vigilante mostra con chiarezza, gli apparati dello Stato persero la partita. Sebbene l'Ond fosse posta sotto la vigilanza del ministero dell'Economia e alle dipendenze del presidente del Consiglio, il partito si impossessò da subito del controllo delle strutture volte ad organizzare la propaganda e il consenso. Al segretario del Pnf fu inoltre conferito l'incarico di commissario dell'ente dal 1927 al 1937 e anche quando si volle porre fine al commissariamento con il tentativo del Ministero delle finanze e del Ministero delle corporazioni (che sostanzialmente ne rivendicava l'esercizio della vigilanza amministrativa) di ricondurre l'Ond nei gangli dell'amministrazione tradizionale, non si ottenne altro che un rafforzamento del potere del partito.

Ciò emerge con maggiore evidenza dal confronto tra la dimensione centrale del fascismo e quella periferica, in cui l'intervento dello Stato doveva spesso fare i conti con le pressioni provenienti dagli interessi politici ed economici locali. L'esempio della realtà lucana è senz'altro paradigmatico poiché aiuta a comprendere — come spiega l'autrice. — "la natura della politicizzazione del Sud e del ruolo particolare di mediazione che le organizzazioni di massa del regime svolsero o non svolsero (con esiti da valutare caso per caso) rispetto alla realtà delle masse meridionali” (p. 160). Il giudizio categorico di "sostanziale fallimento" dell'esperimento dei dopolavoro rurali è in questo senso significativo, sebbene i vantaggi che nel complesso offriva l'adesione agli apparati del nuovo sistema assistenziale consentirono di fatto al regime il mantenimento del controllo sociale e la garanzia di un elevato livello di conformismo politico.

Leonardo Pompeo D’Alessandro

\section{L'antifascismo tra percorsi indivi- duali e storie collettive}

LOREnZo GARDUmi, All'ombra della svastica. La Resistenza nella Zona d'operazioni delle Prealpi: Belluno, Bolzano, Trento 1943-1945, Trento, Fondazione Museo Storico del Trentino, 2015, pp. 351, euro 25.

L'obiettivo del volume appare duplice. Da una parte si cercano, infatti, risposte alla necessità di inquadrare le vicende locali della Resistenza che si sviluppò nei territori della Zona d'operazioni delle Prealpi (Operationszone Alpenvorland Ozav) all'interno delle problematiche proprie della "Resistenze europee". Dall'altra 
si legge la Resistenza come un fenomeno sempre declinato al plurale, riportandola alle sue molteplici dimensioni politiche, militari e umane. Da qui un punto d'osservazione più alto rispetto al passato e un approccio necessariamente più complesso, capace di tenere conto delle più recenti acquisizioni storiografiche.

Il lavoro si articola quindi attorno a una struttura concentrica capace di tenere insieme in un discorso unico dinamiche locali, nazionali e internazionali. Da un'introduzione (pp. 11-16) sul binomio fascismo/antifascismo come fenomeno a livello europeo per quanto articolato in numerose varianti locali e nazionali, si passa ai tre capitoli centrali.

Nel primo (pp. 19-39) viene accennata la molteplicità delle esperienze resistenziali europee innescate dalla presenza dell'invasore tedesco: non solo resistenze armate o esplicitamente politicizzate, ma anche civili e passive, espressione di una più generica e diffusa "resistenza alla guerra". Tutte esperienze plurali, caratterizzate da progettualità per il futuro diverse e spesso confliggenti, che dovettero di volta in volta riadattarsi ai cambiamenti imposti dall'andamento del conflitto e alla morfologia fisica e sociale dei territori in cui si svilupparono. Una pluralità che tuttavia coinvolse anche gli altri attori protagonisti presenti sul palco europeo: nazisti e collaborazionisti a loro volta agirono infatti con strategie sempre differenti, rimodulando violenze e repressioni con il mutare delle condizioni di partenza e in base al comportamento delle popolazioni che subirono l'occupazione.

Il secondo capitolo (pp. 41-75) stringe l'angolo della visuale sul caso italiano, specificando come l'esperienza del fuoriuscitismo, dell'antifascismo europeo e della guerra civile spagnola seppero apportare alla Resistenza italiana un bagaglio di pratiche militari e di conoscenze politiche di cruciale importanza. Il capitolo adotta la periodizzazione ormai canonica proposta a suo tempo da Santo Peli (La Resistenza in Italia. Storia e critica, Torino, Einaudi,
2004), che permette di scandire efficacemente le fasi del confronto politico e militare tra Resistenze, occupanti e collaborazionisti.

Il terzo capitolo (pp. 77-214), indaga come queste dimensioni plurali interagirono all'interno delle tre province inglobate nella Zona d'operazioni, a loro volta caratterizzate da un tessuto sociale discontinuo e fortemente frastagliato sia dal punto di vista politico che nazionale. Al pari della vicina Zona d'operazioni Litorale Adriatico, infatti, le Resistenze all'interno dell'Ozav dovettero confrontarsi con prospettive e termini spaziali definiti dalla presenza di un confine che non solo aveva prodotto con la sua definizione distanze tra le popolazioni locali, per altro reduci dalle politiche fasciste di nazionalizzazione, ma che obbligò le autorità tedesche ad individuare strategie d'intervento capaci di adattarsi ad un quadro potenzialmente esplosivo. L'Ozav diveniva così l'incubatore di esperienze tipico delle "Resistenze di confine", dove appartenenze politiche, nazionali e sociali definirono comportamenti e risposte all'occupazione necessariamente più complesse e diversificate rispetto al restante territorio italiano. Così come avvenne nell'altra Zona d'operazioni, infatti, la dimensione sovranazionale dei problemi affrontati provocò divisioni più profonde tra Resistenze dall'impianto programmatico differente, che impedirono, soprattutto dopo l'inverno 1944-1945 forme di collaborazione durature tra esperienze di diversa ispirazione politico-nazionale e provenienza territoriale.

Gli altri tre capitoli prolungano il monitoraggio delle problematiche sorte già durante la guerra fino al 1948, con l'obiettivo di verificare come le tensioni e i legittimi entusiasmi scaturiti dall'insurrezione generale finirono per stemperarsi in un difficile e discontinuo processo di normalizzazione, reso ancor più insidioso dal difficile riassetto degli equilibri mondiali e dalle dialettiche proprie della Guerra fredda. Un intreccio di questioni e problemi che rende più chiari i motivi che resero l'esperienza resi- 
stenziale un campo di prova difficile per la storiografia, minato da narrazioni agiografiche e da altrettanto radicali rimozioni.

Il lavoro di Gardumi si chiude con una corposa appendice nella quale trova spazio la trascrizione della videointervista a Remo Callone (partigiano della brigata Mazzini, divisione Nino Nannetti), una raccolta di voci biografiche e una cronologia che agevola il lettore nell'inserimento dei fatti salienti avvenuti nell'Ozav all'interno del più vasto panorama nazionale ed internazionale. L'intervista a Callone s'intona agli obiettivi di fondo del volume, rappresentando uno spaccato anti-retorico e umano dell'esperienza resistenziale, in grado di restituire le complessità ambientali e psicologiche a essa connesse.

Complessivamente l'opera si presenta come un aggiornamento storiografico che fa il punto sugli studi degli ultimi quindici anni, scaturiti dalla volontà di ampliare gli orizzonti dei problemi e dei territori presi in considerazione dagli studi sulla Resistenza. Il libro riesce così a offrire una ricostruzione chiara di come l'attività delle molte resistenze nate all'interno dell'Ozav, e le conseguenti azioni repressive naziste e fasciste, abbiano operato all'interno della vasta porzione territoriale che comprendeva tutto l'arco alpino e prealpino orientale. Una chiave di lettura interregionale, peraltro, particolarmente utile dal momento che permette dunque di guardare ai destini delle due Zone d'operazioni come il frutto di una più complessiva gestione strategica dell'intera penisola da parte dei tedeschi, mettendo in connessione vicende territoriali apparentemente distanti, se lette secondo una prospettiva localistica.

Irene Bolzon

Emilio Pianezzola, Concetto Marchesi. Gli anni della lotta, Padova, Il Poligrafo, 2015, pp. 101, euro 18.

A oltre mezzo secolo dalla scomparsa, su Concetto Marchesi - docente universitario, iniziatore della Resistenza in
Veneto, padre costituente e uomo politico - non possediamo ancora uno studio storico-biografico che renda conto compiutamente del suo percorso di vita, umano e politico. Un generoso tentativo in questa direzione fu compiuto nel centenario della nascita da Ezio Franceschini (Concetto Marchesi. Linee per l'interpretazione di un uomo inquieto, Padova, Antenore, 1978), dopo che per oltre un ventennio si erano susseguiti, su quotidiani e riviste, saggi di taglio prevalentemente commemorativo e cronachistico, o, al più, esegetico della produzione letteraria del professore siciliano. Il volume, però, pur presentando una cospicua documentazione inedita, risentiva della comprensibile difficoltà di Franceschini, insigne studioso di letteratura latina medievale, a disincagliarsi dalla condizione di amico fraterno, fedele compagno di lotta e "discepolo" di Concetto Marchesi, oltre che di osservatore partecipe della gran parte delle vicende narrate. Mosso da esigenze celebrative, non ha posto rimedio a questa carenza, grave e curiosa a un tempo - se si conviene che Marchesi sia stato tra gli intellettuali comunisti di più profondo acume -, nemmeno il libro curato da Giuseppe Zaccaria, Concetto Marchesi e l'Università di Padova 1943-2003 (Padova, Cleup, 2004), che raccoglieva gli interventi pronunciati nel corso del convegno $A$ sessant'anni dall'Appello agli studenti di Concetto Marchesi, di cui, comunque, è d'obbligo segnalare il denso saggio di N. Bobbio, Profilo di Concetto Marchesi, (pp. 73-80), già pubblicato nel suo La mia Italia, curato da P. Polito (Firenze, Passigli, 2000, pp. 195-203).

Non è nelle intenzioni di Pianezzola, professore emerito di letteratura latina nell'ateneo patavino, proporre un profilo organico di Marchesi. Il breve volume, nondimeno, malgrado non contempli riferimenti a una documentazione inedita, ha il merito di passare al vaglio una pluralità di fonti letterarie, allo scopo di focalizzare l'attenzione sulle circostanze più significative della vita dell'illustre collega nel 1943, 
"l'anno grande della sua vita" (M. Isnenghi, Presentazione, p. 8), quando l'incalzare degli eventi, tra l'estate e l'autunno, gli impone di divenire protagonista della vicenda italiana.

Non era sempre stato così. Durante il ventennio Marchesi, pur non facendo mai mistero del suo credo politico, aveva scelto di non contrapporsi frontalmente al regime, ma di eroderne dall'interno le granitiche certezze su cui poggiava, "suggerendo a chi lo ascoltava o leggeva spunti inequivocabili di riflessione sulla libertà e sull'eguaglianza sociale di una vera democrazia“" (p. 27). Con questo spirito, e non certo per adesione al fascismo, nel 1931 - confortato anche dal parere di Togliatti (G. Amendola, Lettere a Milano. Ricordi e documenti 1939-1945, Roma, Editori Riuniti, 1973, p. 143) - non si era sottratto al giuramento imposto da Mussolini ai docenti universitari.

La sfiducia del Gran Consiglio al Duce determinò un cambio di passo nella sua azione: non più — non solo - impegno intellettuale, ma militanza concreta, pratica politica. Già in maggio Marchesi aveva operato per stringere collegamenti con gli ambienti monarchici e le gerarchie militari, incontrando tra gli altri il generale Raffaele Cadorna e Maria José; successivamente, il 12 agosto, con Giorgio Amendola e Giovanni Roveda rappresentò il Pci nella riunione del Comitato centrale dei partiti antifascisti, caldeggiando vivamente l'ipotesi insurrezionale.

Cambiarono le forme dell'impegno, dunque, ma restò immutato il suo nucleo ideale: è questa, in fondo, la tesi che attraversa il libro, sorretta dal riscontro fornito dagli scritti e dai documenti pubblicati da Franceschini (Concetto Marchesi cit., pp. 155-360), e argomentata attraverso l'esame attento dei principali interventi pubblici di Marchesi in quei concitati mesi (riprodotti in appendice, pp. 63-97). Per esempio, nel Discorso per l'inaugurazione del $722^{\circ}$ anno accademico dell'ateneo padovano, il 9 novembre 1943, interrotto da un gruppo di militi fascisti che "rompe- va la tregua ostile fra università e potere esterno“ (p. 45), come nell'Appello rivolto agli studenti il $1^{\circ}$ dicembre, il giorno dopo aver consegnato le dimissioni da rettore al ministro dell'Educazione nazionale Biggini (p. 73), l'autore rintraccia motivi espressi da Marchesi in altri frangenti - i due volumi della Storia della letteratura latina (Messina-Roma, Principato, 1925 e 1927) — o, addirittura, in altre "epoche" — il volumetto di poesie Battaglie (Catania, Tipografia dell'Etna, 1896) — , rilevando una sorprendente "continuità di immagini e di atteggiamento interiore, di fronte a cui sono cambiati - forse solo apparentemente - l'interlocutore e l'avversario" (p. 51).

Fa da sfondo al rapido succedersi degli eventi Padova, "la città diletta" (C. Marchesi, M. Valgimigli, Lettere a una libreria, I. De Luca, A. Zadro (a cura di), Padova, G. Randi, 1968, p. 4), e soprattutto la sua università, che di quella costituisce 1'“asilo di studi", il "porto in mezzo alla bufera" (Discorso, p. 66). Due luoghi, due spazi fisici ma non immobili che, anzi, interagiscono con i grandi quadri che vengono determinandosi e "rispondono" alle sollecitazioni del rettore: sono gli studenti, infatti, che il giorno dell'inaugurazione dell'anno accademico travolgono con "l'immensa ondata" di "infrenabile sdegno" il manipolo di fascisti "[...] sciagurati, violatori dell'Aula Magna" (Appel$l o$, p. 74), e molti di loro figureranno tra quei centosedici caduti nella Resistenza per cui l'ateneo meriterà la Medaglia d'Oro al Valor Militare (Il Bo Medaglia d'Oro al Valor Militare, in appendice a Concetto Marchesi e l'Università di Padova cit., p. 92).

Carmelo Albanese

Giovanna Caleffi Berneri, Un seme sotto la neve. Carteggi e scritti. Dall'antifascismo in esilio alla sinistra eretica del dopoguerra (1937-1962), a cura e con introduzione di Carlo De Maria, prefazione di Giampietro Berti, nota conclusiva di Goffredo Fofi, Reggio Emilia, Biblioteca 
Panizzi, Archivio Famiglia Berneri e Aurelio Chessa, 2010, euro 10.

La lettura di questo libro ci consente di ripercorrere, da un'angolazione alquanto singolare, eventi e figure della storia politica e culturale italiana tra la vigilia del secondo conflitto mondiale, la guerra e il primo quindicennio di esperienza repubblicana. Un quarto di secolo, 19371962, dallo scontro civile in Spagna, che fu preludio alla guerra, agli anni del boom economico e al tempo stesso di molteplici contraddizioni politiche in Italia, scorre pertanto davanti ai nostri occhi così come lo ha vissuto, e come ce lo fa rivivere, una figura fino a oggi poco conosciuta, ma che al termine della lettura si lascia con il senso di un'acquisita confidenza. Giovanna Caleffi nel libro ci appare in veste di testimone e di interprete di un'epoca che corrisponde a quell'arco cronologico, ma che già all'inizio di essa aveva alle spalle un intenso e tragico passato, decisivo a orientarne la successiva azione e il pensiero. Dal gennaio 1917 e fino al 1937, anno quest'ultimo che costituisce il termine $a$ quo del volume: Giovanna Caleffi era stata, infatti, la moglie dell'intellettuale e militante anarchico Camillo Berneri; i due si erano sposati dopo che lei aveva conseguito il diploma di maestra elementare, quando entrambi non erano ancora ventenni e Camillo fu assassinato per mano comunista in Spagna appunto nel maggio 1937, a Barcellona ove era accorso a combattere sul fronte antifranchista e repubblicano. Giovanna Caleffi aveva condiviso con Berneri frequentazioni intellettuali negli anni degli studi (prima fra tutti quella con Gaetano Salvemini, professore di Camillo nella facoltà di Lettere dell'ateneo fiorentino) e poi, all'avvento del fascismo, intimidazioni e violenze, l'esilio in Francia e, oltre alle amarezze e alle illusioni della politica, le relative, e a tratti angoscianti ristrettezze dell'economia familiare.

L'uccisione di Berneri costituisce non solo l'antefatto, ma anche il momento genetico di tutta l'appassionante vicenda in- tellettuale e umana documentata dal libro. Ed è scelta suggestiva del curatore offrire come primo testo alla nostra attenzione la lettera di condoglianze che dalla lontana Montevideo scrive a Giovanna, Luce Fabbri, figlia di uno dei maggiori esponenti dell'anarchismo italiano ed ella stessa scrittrice e militante anarchica (una delle tante significative figure che la lettura del libro invita quasi a ogni passo a approfondire): "una notizia terribile ci è stata portata dai giornali [...] Forse lei saprà contro chi va diretto questo sentimento d'orrore, che in certi momenti arriva a essere odio"; non senza tradire un comprensibile imbarazzo per il carattere "scandaloso" di quell'evento (tale da segnare a lungo la memoria dell'assassinio di Berneri e, se è consentito l'accostamento, non privo di analogie con certi giudizi relativi a eventi terroristici in Italia negli anni Settanta circa i loro "sedicenti" esecutori). Alle figlie di Berneri - scriveva in quella commovente pagina la giovane compagna di ideali anarchici dal proprio esilio in Sudamerica - si poteva ben dire che "il loro babbo" era "caduto per conquistare a tutti [un'] isola di pace [...] caduto nella lotta contro il fascismo. A qualunque partito appartengano quelli che l'hanno ucciso sono fascisti. Sono i nemici di sempre" (pp. 3-4).

Se la vita di Giovanna Caleffi si era svolta fino a quel momento sotto il segno di una partecipazione alle idee del marito, che si esprimeva però soprattutto nella cura delle numerose attività familiari e domestiche (erano nate, tra 1918 e 1919, due bambine), poi anche della gestione di una piccola attività commerciale (prodotti alimentari italiani) alla periferia di Parigi, dal momento dell'uccisione di Camillo, lei avrebbe trovato ragione di vita nell'assumere il testimone di quella battaglia, in tutte le sue implicazioni: ideali, relazionali, ed anche - a partire dalle radicalmente mutate condizioni nell'Italia del dopoguerra (dopo aver patito la prigionia prima nella Francia occupata e poi in Germania, infine il confino in provincia di Avellino) — organizzative e pratiche. 
Il libro presenta una struttura tripartita. A un ampio saggio introduttivo di Carlo De Maria (del quale va segnalata una precedente, densa ricostruzione della vita e della personalità intellettuale di Berneri: Camillo Berneri. Tra anarchismo e liberalismo, Milano, Angeli, 2004, cui i lettori interessati alla suggestiva figura di Giovanna Caleffi potranno ricorrere come a una importante lettura complementare) seguono due vaste sezioni, di pressoché eguale dimensione: la prima (pp. 1-310) dedicata ai Carteggi; la seconda (pp. 311-578) agli Scritti, entrambi presentati in un ordine cronologico interno. Si tratta di una scelta estremamente felice: le lettere, che danno anche largo spazio agli interlocutori di Giovanna Caleffi, rimandano alle pagine da lei successivamente o contestualmente edite; così come gli scritti pubblicati, a partire dal 1944, specularmente e costantemente rimandano al ricco, e internamente assai variegato, corpus epistolare.

E del resto ciò corrisponde a una scelta metodologica precisa, che, come sottolinea De Maria, consente di “'fare la spola' tra pubblico e privato, con rimandi frequenti tra le corrispondenze epistolari e l'attività giornalistica [...] Solo in questo modo, infatti, è possibile restituire al lettore la trama dei rapporti che intercorrono tra affetti familiari, reti amicali e attività pubblica" (p. XIII). A questa prospettiva - che il curatore fa risalire al "suggerimento metodologico formulato, all'inizio degli anni novanta da Mariuccia Salvati a orientare gli studi verso la 'contrapposizione pubblico/privato"” (p. XIV) - la figura di Giovanna Caleffi Berneri corrisponde in modo pieno e suggestivo. La valorizzazione, caratteristica del pensiero anarchico, dei piccoli gruppi minoritari, sembra qui declinarsi nella figura collegiale, e protagonistica, del piccolo gruppo familiare, in cui anche coloro che muoiono continuano a svolgere un proprio ruolo, continuano a partecipare al movimento delle idee: $\mathrm{Ca}$ millo, certamente, attraverso i suoi scritti e in quanto vittima illustre delle ideologie totalitarie. Ma anche Marie Louise - scomparsa poco più che trentenne e continuatrice intellettuale dell'opera del padre, animatrice del movimento anarchico in Inghilterra - dopo il 1949 continua a vivere per iniziativa della madre attraverso la colonia Maria Luisa Berneri, una coraggiosa iniziativa nel campo sociale e al tempo stesso di grande valore pedagogico. La famiglia nucleare, istituzione portante della società borghese tra la fine del XIX secolo a tutto il XX, e della quale il declino e forse addirittura la liquidazione appare a noi in atto - a livello di costume ed anche legislativo - , ha per tutto il libro un'importanza vitale. Le figlie di Giovanna e di Camillo Berneri, oltre alla già citata Marie Louise, Giliana, che resterà a Parigi ed eserciterà la professione di medico, la stessa "mamma di Camillo", Adalgisa Fochi insegnante che aveva avuto Giovanna allieva alle scuole magistrali e che l'aveva presentato al figlio, del quale poi serberà la memoria anche con un opuscolo scritto negli ultimi anni (Con te, figlio mio!), sono una presenza costante dell'epistolario: e mai tra la dimensione privata e quella dell'impegno civile ve n'è una che sia prevaricatrice e annullatrice dell'altra.

Il mondo delle memorie e degli affetti familiari e privati è tuttavia destinato ad allargarsi nella prospettiva e nell'intendimento di agire - in chiave di radicale e a tratti dichiaratamente utopica trasformazione - sulla società intera (e in modo particolare, nel caso specifico, sulla società nazionale italiana: di nazional-anarchismo aveva del resto in modo esplicito parlato Camillo Berneri). Dalla fine del 1944, Giovanna Caleffi si stabilì a Napoli, prima nella parte d'Italia liberata e sottoposta a controllo alleato, poi nell'Italia repubblicana, dove inizia un'intensa attività pubblicistica, soprattutto attraverso la rivista "Volontà" da lei fondata e diretta assieme a Cesare Zaccaria, acuta e complessa figura di intellettuale anarchico, amico a suo tempo di Camillo Berneri e ora divenuto, in una linea di ideale, dichiarata continuità, il suo compagno di vita. È qui possibile soltanto accennare alla ricca galleria 
di interlocutori celebri o meno conosciuti che dalla corrispondenza alle pagine edite (tra cui, negli ultimi anni, "Il lavoro nuovo" di Genova diretto da Sandro Pertini) sostanziano la trama di relazioni amicali e intellettuali (sovente le due cose assieme) di Caleffi. Sta a sé il rapporto con Salvemini, "in qualche modo protettore, sostituto di padre" (così Goffredo Fofi nella nota conclusiva) con il quale Giovanna avrebbe sempre coltivato un rapporto tanto rispettoso quanto di assoluta franchezza, suggellato da un bellissimo articolo da lei scritto all'indomani della scomparsa di colui che era stato amico e maestro suo e di Camillo. E ancora vanno citati i nomi di Ignazio Silone, Piero Calamandrei, Ernesto Rossi (Caleffi collaborerà anche con alcuni articoli a "Il Mondo"), Angelo Tasca, Aldo Capitini, Danilo Dolci, Adriano Olivetti. E c'è anche, proprio nell'anno del Nobel e a seguito di un loro incontro a Parigi, una lettera di Albert Camus (da lei assieme a Silone considerato maestro di letteratura libertaria).

La posizione di Caleffi nel panorama della cultura italiana dell'immediato dopoguerra e nel corso di tutti gli anni Cinquanta è, appropriatamente, da De Maria ascritta a un filone "ereticale" del quale, all'inizio del saggio introduttivo, si osserva non senza preoccupazione, l'inaridirsi ai nostri giorni. Il giudizio sulla classe di governo è caratterizzato da accenti radicalmente critici e polemici in una certa affinità con l'interpretazione cosiddetta azionista della prima fase di vita repubblicana: il "governo dei preti" sembra avere semplicemente "rimpiazzato" il fascismo e il partito democristiano appare come il mero esecutore di una volontà clericale, della "gelida frenesia di potere della chiesa cattolica", fino a configurare una "nuova tirannia" (p. 125, in nota), una sorta di "totalitarismo cattolico" (dicembre 1952, p. 403). La diffidenza o la ripulsa anarchica nei confronti di partiti, chiese, organizzazioni in genere non escluse quelle sindacali (il sindacato post-bellico "non differi[va]" ai suoi occhi "da quello fasci- sta", p. 85) ricorre con insistenza nelle pagine di Caleffi e non risparmia la maggior forza di opposizione politica in Italia. Anzi, la polemica anticomunista assume accenti di particolare vigore: sia sul piano del giudizio storico e teorico ("Stalin è la conclusione logica di Marx e di Lenin": p. 463), che in relazione ai metodi adottati. Anche dopo il XX congresso e i fatti di Ungheria, Togliatti e i suoi subalterni continuavano ad agire "al di fuori di ogni principio morale" (p. 227, in nota): "Ai capi ed ai dirigenti comunisti non si può perdonare che nell'interesse del partito si servano della menzogna, della calunnia e se necessario dell'assassinio" (così scriveva colei che era stata la moglie di Camillo Berneri: Conversazione con Ignazio Silone, maggio 1957, p. 483).

A causa anche della scarsa influenza delle formazioni laiche minori, sembrava non arginabile l'affermarsi di "un totalitarismo invadente, sia nero che rosso" (lettera a Silone, 14 aprile 1957, p. 226). A tale realtà venivano a contrapporsi battaglie civili come quella per una politica (dichiaratamente neomalthusiana) di limitazione delle nascite, che la legislazione di epoca fascista, ispirata a opposti principi demografici, a norma di legge sanzionava. Se indubbio era il valore di libertà legato a quella battaglia, peraltro coronata dalla doppia assoluzione nei processi di Napoli e poi di Milano, al lettore di oggi essa dà anche il senso di una grande distanza: se era allora possibile scrivere che a "una popolazione eccedente di dieci o quindici milioni individui, in aumento di circa mezzo milione l'anno" era legato "il cronico problema della miseria dei lavoratori italiani" (cfr. p. 326), oggi, alle prese con problemi demografici di segno diverso, quelle controversie invitano, evidentemente, a una riflessione complessa sulle dinamiche di sviluppo socio-economico, e al tempo stesso dei costumi, nell'Italia del XX e XXI secolo.

La vita nazionale degli anni Cinquanta appariva, ai redattori di una rivista come "Volontà" e nell'ambito peraltro di una 
più vasta corrente di opinione, come presa "nella morsa delle due chiese" (per usare l'espressione di Giampiero Berti nella prefazione al volume), "avvelenata da ubbidienze di ogni tipo e colore" (lettera ad Alberto Mondadori, $1^{\circ}$ dicembre 1953, p. 197), sopraffatta da quel conformismo e da quelle inclinazioni gregarie che lo spirito anarchico di Giovanna Caleffi e le tradizioni della famiglia intellettuale cui essa apparteneva, vedevano come il male supremo.

Se l'invettiva si addice agli eretici e un certo pessimismo, dai toni anche radicali risulta funzionale alla critica, il tono necessariamente ma anche insistentemente polemico di quelle battaglie richiede, a distanza e dal punto di vista del lettore di oggi, altrettanto necessarie contestualizzazioni e mediazioni. Il timore che il volume - oltretutto di così ampie dimensioni — potesse risultare a tratti faticoso a causa di un certo carattere di monotonia o di quella mancanza di colori in modo quasi inevitabile legati all'attività militante, poteva insomma corrispondere a un timore legittimo.

Non è così per fortuna; o non tanto per fortuna, quanto per merito proprio di Giovanna Caleffi. Dalle pagine di questa doppia antologia, dalle lettere così come dagli articoli, emerge una personalità originale, in cui a una modestia che avvertiamo sincera si unisce una forza di argomentazione vigorosa e di un'accattivante naïveté. Nel procedere della lettura si ha come la percezione sia di una naturale capacità espressiva, sia di un talento letterario che, da sempre privo di ostentazioni e di retorica, si andava col tempo progressivamente affinando. Ne sono testimonianza alcuni articoli degli ultimi anni: da quello dedicato alla città in cui aveva tanti anni abitato Napoli a occhio nudo. Grattacieli e tuguri, apparso su "Il Mondo" nel dicembre 1959) fino alla riflessione densa di suggestioni sociologiche e storiche (vero e proprio atto di amore nei confronti del suo luogo natale, il comune di Gualtieri in provincia di Reggio Emilia), dal titolo I paesi si trasformano ("Volontà", novembre 1960, pp. 561-567). Ma anche, sul versante epistolare, le poche righe, esemplari di sobrietà e di dignità, con cui Giovanna Caleffi dava conto della fine del sodalizio sentimentale e politico con Cesare Zaccaria (lettera ad A. Bortolotti 20 giugno 1957, p.235) con conseguenze anche sull'attività della Colonia, per il venir meno della disponibilità della villa presso Salerno di proprietà di Zaccaria. "Come accade di tutti i doni che vengono elargiti dall'alto" e che "si perdono facilmente", avrebbe osservato Giovanna Caleffi (p. 575), in un'estrema (è l'ultimo testo che l'antologia presenta) e senza dubbio amara ma anche serenamente realistica declinazione di quella spola tra vita privata e dimensione pubblica che lo studio della concretezza storica invita costantemente a riconoscere e a comprendere.

Mario Tesini

\section{"Non di solo pane": il miracolo eco- nomico}

Emanuele Bernardi, Il mais "miracoloso”. Storia di un'innovazione tra politica, economia e religione, Roma, Carocci, 2014, pp. 199, euro 22.

Il libro ricostruisce la storia dell'introduzione in Italia nel secondo dopoguerra dei mais ibridi americani, un'innovazione tecnico-scientifica destinata negli anni successivi a modificare in profondità l'agricoltura e l'alimentazione. Il miglioramento genetico del mais - ottenuto attraverso l'incrocio di tipi diversi autofecondati - risaliva ai primi del Novecento e si era diffuso rapidamente negli Stati Uniti durante il New Deal; le piante prodotte da sementi ibride presentavano caratteri di omogeneità, di vigoria, di dimensioni superiori rispetto al passato e consentivano perciò coltivazioni più razionali e una maggiore produttività. L'intento dell'autore è stato quello di esaminare le implicazioni politiche, economiche, cul- 
turali e ambientali connesse all'adozione di questa nuova tecnologia nel contesto del progressivo delinearsi della Guerra fredda.

Una sedimentata storiografia ha studiato il Piano Marshall e come il trasferimento di know how sviluppato oltre oceano nell'Europa postbellica abbia costituito un importante strumento di affermazione dell'egemonia americana in campo politico ed economico. La ricerca di Bernardi ne offre un'ulteriore dimostrazione da una prospettiva inedita e italiana (anche se non mancano cenni comparativi ad altri paesi, in primis la Francia). Nel caso dell'agricoltura, la convergenza tecnologica sulle sementi ibride rientrava in una strategia più generale mirante a promuovere una radicale modernizzazione del settore che, migliorando le condizioni di vita nelle campagne, contribuisse a contenere la forte conflittualità politica e sociale. Non diversamente dalla coeva riforma agraria il nuovo cereale rispondeva a questa funzione: incrementava produttività e quantità, promettendo risultati "miracolosi" sia per i grandi sia per i medi e piccoli produttori.

Per sostenere l'affermazione dei mais ibridi in Italia non furono lesinati sforzi: dalla propaganda attraverso manifesti, opuscoli e cinegiornali all'occasione offerta dall'alluvione del Polesine nel 1951, l'impegno americano fu intenso e proseguì oltre la fine del Piano Marshall. Sulle autorità e sulle istituzioni tecnicoscientifiche italiane furono esercitate notevoli pressioni tanto da parte del governo di Washington quanto dalle lobby dei produttori di sementi ibride. Tuttavia l'autore non interpreta in senso eterodiretto la scelta netta compiuta dall'Italia ed evidenzia invece gli elementi di convergenza attiva. Sul piano tecnico e scientifico si distinse l'azione della Stazione sperimentale di maiscoltura di Bergamo, che sotto la guida di Luigi Fenaroli divenne, spiega Bernardi, l'epicentro della trasformazione dell'agricoltura italiana avviata dai mais ibridi. A livello politico la convergenza dell'Italia poggiava sulla condivisione di obiettivi comuni con il nuovo alleato: sta- bilizzazione, modernizzazione e contrasto del pericolo comunista. La scelta dei mais ibridi costituì il tassello di un più generale processo di rinnovamento dell'apparato produttivo che, con la fine dell'autarchia, andò di pari passo con l'apertura ai commerci internazionali; rispetto a questo secondo aspetto Bernardi ne rileva l'asimmetria, perché all'apertura del mercato interno alla concorrenza estera - come appunto nel caso dei mais ibridi - non corrispose un reciproco trattamento per i prodotti agricoli italiani.

Nella battaglia per i mais ibridi Bernardi attribuisce un ruolo particolare alla Chiesa di Pio XII. Per motivi di ordine politico e umanitario il Vaticano condivise il progetto dei mais ibridi e, particolarmente durante il 1950, anno giubilare, fornì un valido sostegno: sul piano materiale, mobilitò la propria rete assistenziale a livello nazionale e transnazionale con il compito di favorire raccolta e distribuzione; contribuì inoltre a livello simbolico e religioso, legittimando pubblicamente le nuove sementi. Alla lunga tuttavia la convergenza trovò un limite nelle diverse priorità e finalità perseguite: assistenzialismo sociale e pacifismo per la Chiesa, produttivismo militarista per gli Stati Uniti dopo la guerra di Corea.

Nel libro trovano spazio le voci e le ragioni di quanti si opposero ai mais ibridi. In sede politica si distinse la sinistra e in particolare il Partito comunista, che si mobilitò in Parlamento e nei territori. La posizione del Pci derivava da diversi fattori: la preferenza accordata al sistema sovietico, ritenuto un'alternativa tecnicoscientifica superiore; la convinzione che i problemi di produttività dell'agricoltura fossero risolvibili con una radicale riforma agraria;i timori perla subordinazione dell'Italia agli interessi politici ed economici americani. Pur rimanendo un aspetto poco indagato, sembrano comunque emergere sfumature e una certa articolazione all'interno dell'intransigentismo comunista. Sarebbe stato inoltre interessante approfondire qualche caso specifico, inizian- 
do dall'Emilia, per comprendere meglio le dinamiche intercorse fra la propaganda comunista e l'atteggiamento dei contadini.

Accanto all'opposizione politica, Bernardi riferisce la diffidenza e, a volte, l'ostilità diffuse fra i coltivatori e non pochi tecnici e accademici. Non si trattava - come semplicisticamente ritenevano gli americani — solo di "conservatorismo", ma di una critica che scaturiva da dati fattuali: i semi d'oltreoceano non si rivelarono sempre e dovunque "miracolosi", come era stato promesso. I risultati della sperimentazione condotta nel 1948-1949 furono controversi: in vari casi i mais ibridi segnarono rese inferiori alle sementi locali e inoltre si rivelarono soggetti a malattie e parassiti da cui le varietà "indigene" erano immuni o quasi. Un altro fattore che all'inizio frenò l'avanzata delle sementi ibride fu il prezzo elevato, anche perché si potevano utilizzare una sola volta - pena un "crollo" in caso di seconda semina - e comportavano costi aggiuntivi per i particolari trattamenti di cui necessitavano. Per ovviare al problema si fece largo ricorso a sussidi pubblici e donazioni, una soluzione che, come sottolinea l'autore,produsse alla lunga effetti distorsivi sul mercato. Ciò nonostante l'avanzata dei mais ibridi fu inarrestabile: la superficie coltivata in Italia passò dai 1.500 ettari del 1948 a 371.000 ettari di fine anni Cinquanta. La diffusione non fu però omogenea a livello territoriale, facendo segnare differenze marcate fra macro-aree (maggiore diffusione al Nord, limitato sviluppo nel Sud) e all'interno delle stesse (per esempio, fra Lombardia e Veneto).

A fronte del successo planetario dei mais ibridi rimane sotto vari punti di vista il senso di una perdita. L'autore ricorda a più riprese le conseguenze della trasformazione su consuetudini e pratiche consolidate, ponendo l'accento in particolare sulla scomparsa di un patrimonio colturale e culturale sedimentatosi nel tempo: è lungo l'elenco di varietà maidicole italiane destinate a sparire oppure a drastici ridimensionamenti. Con esse scomparvero - per obsolescenza tecnologica - anche antiche pratiche e conoscenze; il mutamento investì,infatti, la professionalità degli agricoltori nel senso - sottolinea Bernardi - di una loro dequalificazione. La parte finale si focalizza sugli anni più recenti, stimolando riflessioni su tematiche di stretta attualità: dalla sicurezza alimentare agli organismi geneticamente modificati, dal rapporto fra produttori e consumatori alla sostenibilità ambientale dello sviluppo in agricoltura. Si tratta - come sottolinea Bernardi — di questioni aperte che, oggi come ieri, generano discussioni e divisioni profonde a livello sia politico che di opinione pubblica, investendo le fondamenta del rapporto fra uomo, alimentazione e ambiente.

Stefano Mangullo

Damiano Garofalo, Vanessa Roghi (a cura di), Televisione. Storia, immaginario, memoria, Soveria Mannelli, Rubettino, 2015, pp. 270, euro 16.

Il volume raccoglie una serie di contributi e interventi che fanno il punto sulla storia del sistema televisivo in Italia, a partire dalle origini (1954) e sul suo impatto di reciproco condizionamento sulla società, i costumi, la cultura, l'educazione.

Attraverso una struttura tripartita emerge un discorso complesso e incrociato in cui le linee storiche si intrecciano con i più insidiosi temi dell'immaginario e della memoria, a partire dall'assunto che la storia della televisione e delle modalità con cui è stata consumata e introiettata può essere "parte di una storia del XX secolo", e va considerata in funzione di una esplorazione "di lunga durata".

Nella sezione "Storia", attraverso i quattro saggi di Enrico Menduni, Mariagrazia Fanchi, Andrea Sangiovanni e Luca Barra, possiamo ripercorrere alcuni momenti: l'era della "veterotelevisione" caratterizzata da una tecnologia primitiva, una diffusione ridottissima e poi rapidamente capace di crescita esponenziale, un mondo 
naif ed elementare, in cui dominano Mike Buongiorno e il modello americano, molto abilmente declinato all'italiana e in versione casalinga. Sono anni in cui la televisione compie una sua funzione unificatrice nel senso della produzione di una "cultura media" della popolazione italiana, uscita dalla guerra, scarsamente scolarizzata, divisa linguisticamentee appunto sul piano culturale complessivo.

Che in questi anni sia forte l'imprinting cattolico lo documenta il contributo di Maria Grazia Fanchi con una analisi puntuale dell'interesse che il mondo cattolico, anche quello degli intellettuali, manifesta per la televisione; la televisione è individuato come uno strumento di primo ordine per "evangelizzare", ma anche per acculturare e educare le masse.

Le profonde mutazioni che si producono a partire dal 1974 con l'affermarsi di un sistema televisivo misto pubblico/privato, che diventa poi dal 1984 un vero e proprio duopolio, costituiscono il focus di indagine del saggio di Andrea Sangiovanni. I primi anni Settanta sono anni in cui si fa sentire l'esperienza sessantottina, l'aspirazione a una "riappropriazione dal basso degli strumenti d'informazione". L'utopia e l'anarchia durano poco, ben presto travolte da rapide trasformazioni tecnologiche e soprattutto fagocitate da chi riesce imporre in modo totalizzante il proprio modello comunicativo, Berlusconi e Mediaset. Non si tratta naturalmente solo di un evento di carattere industriale ma corrisponde a una profonda mutazione nel pubblico, negli spettatori, uno degli aspetti essenziali del riflusso degli anni Ottanta, il tutto però condito dall'irruzione della pubblicità assunta in dosi massicce dal mondo di Mediaset e ben presto anche accolta nei programmi Rai. I primi anni Ottanta sono anni di battaglia che si concluderanno nel 1984 con il decreto Craxi e la definitiva spartizione del sistema televisivo tra Rai e Mediaset.

In questo quadro è fondamentale la definizione e strutturazione del "palinsesto", analizzato nel contributo di Luca Barra, che ne precisa, attraverso una puntuale periodizzazione, le funzioni in relazione al pubblico e al suo variare, fino agli anni Ottanta quando "il palinsesto da artigianato diventa scienza", un sistema rigoroso e consolidato di mandare in onda i programmi e di attingere con economia al magazzino. E intanto a partire dalla metà degli anni novanta con l'arrivo dei canali tematici gli equilibri cambiano di nuovo, ma queste sono poi nuove storie da raccontare, ancora tutte in divenire.

Nella sezione "Immaginario" Giuliana C. Galvagno dimostra quanto siano stati importanti, per la costruzione di un'identità nazionale negli anni compresi tra il 1954 e il 1961, nel paese che usciva dalla guerra, gli sceneggiati, a cui va anche il merito di un primo riuscito tentativo di costruire un linguaggio specifico della televisione. Segue dal 1961 al 1994 la stagione dei programmi di storia analizzati da Vanessa Roghi: finita la celebrazione risorgimentale, saturato lo sceneggiato e con i progressi della tecnologia che rende possibile l'uso di materiale d'archivio filmato, inizia l'era dei programmi di storia. Alla metà degli anni Sessanta, a vent'anni dalla fine della guerra, si può cominciare a parlare di nazismo e di fascismo, temi tabù fino a quel momento e se ne possono proporre approfondimenti e interpretazioni critiche, nonostante le memorie degli italiani su questi temi restino divise. E il periodo in cui si elaborano anche forme innovative e sperimentali di linguaggio come nel caso del programma di Zavoli del 1972 Nascita di una dittatura, che introduce anche l'uso dei testimoni.

A partire dagli anni novanta la storia incontra la fiction, come ci viene presentato nel contributo di Monica Jansen e Maria Grazia Bonaria Urban. La fiction, nella modalità delle miniserie, diventa uno strumento di interpretazioni revisionistiche, inducendo nello spettatore quello che le autrici chiamano "l'errore presentista", vale a dire la proiezione sugli eventi storici delle urgenze del presente, creando le condizioni per l'elaborazione di una me- 
moria storica consolidata ma spesso falsata. E non mancano naturalmente le serie o le fiction dedicate all'attualità, dagli anni di piombo, alla mafia, fino a giungere alla saga di Romanzo Criminale, oggetto di una specifica analisi da parte di Guido Vitiello, che definisce "circo mediatico-letterario" la spirale in cui si avvolge o involve il consumo di un determinato evento della cronaca o della storia, una volta che esso sia stato mediatizzato.

Il capitolo "Memoria" è quello per certi versi più scivoloso nel tentativo di mettere a fuoco le procedure e le modalità con cui il consumo televisivo crea una memoria collettiva. Damiano Garofalo utilizza stralci di alcuni diari, dell'archivio di Pieve Santo Stefano, per delineare il consumo di tempo libero tra radio, televisione, cinema e qualche sprazzo di vita sociale nei giovani di estrazione popolare tra la fine degli anni Cinquanta e gli anni Settanta. E il consumo televisivo in quegli anni e con quei giovani è ancora assai limitato. Ma nella generazione successiva, quella che potremo chiamare la generazione dei "nativi televisivi", Piermarco Aroldi documenta, anche attraverso l'uso di interviste mirate e di focus group, come la ricezione televisiva, divenuta quotidiana e quantitativamente ben più massiccia, si intrecci in un nodo inscindibile coi ricordi personali dell'infanzia.

E così avviene che gli eventi della storia, attraverso la comunicazione televisiva, si sedimentino nella biografia personale: è un processo ben analizzato da Olimpia Affuso, grazie a un ampio lavoro di ricerca, che ricostruisce come si generi una memoria collettiva in cui i fatti sono gerarchizzati, interpretati, rinarrati e siano destinati a consolidarsi in giudizi e interpretazioni spesso falsi ma difficilmente scalfibili.

Infine Emiliano Perra propone - a partire da una miniserie dedicata al conflitto israelo-palestinese The Promise, trasmessa in Inghilterra nel 2011 - il caso di studio di un programma che, suscitando un dibattito intenso, a volte feroce, è riuscito ad affrontare, in modo diretto e critico, una vicenda controversa della storia nazionale.

In appendice le conversazioni con Francesco Casetti e Mario Morcellini, che hanno percorso un lungo tratto di studi sulla televisione a partire proprio dalla loro fondazione, riassumono in una efficace sintesi il lavoro fatto e quello che si potrà fare per costruire una storia, work in progress, della televisione e del suo impatto sulla mentalità collettiva.

Paola Olivetti 